\title{
A survey of Calabi-Yau manifolds
}

\author{
Shing-Tung Yau
}

\section{CONTEnts}

1. Introduction 278

2. General constructions of complete Ricci-flat metrics in Kähler geometry

2.1. The Ricci tensor of Calabi-Yau manifolds 278

2.2. The Calabi conjecture 279

2.3. Yau's theorem 279

2.4. Calabi-Yau manifolds and Calabi-Yau metrics 280

2.5. Examples of compact Calabi-Yau manifolds 281

2.6. Noncompact Calabi-Yau manifolds 282

2.7. Calabi-Yau cones: Sasaki-Einstein manifolds 283

2.8. The balanced condition on Calabi-Yau metrics 284

3. Moduli and arithmetic of Calabi-Yau manifolds 285

3.1. Moduli of K3 surfaces 285

3.2. Moduli of high dimensional Calabi-Yau manifolds 286

3.3. The modularity of Calabi-Yau threefolds over $\mathbb{Q} \quad 287$

4. Calabi-Yau manifolds in physics 288

4.1. Calabi-Yau manifolds in string theory 288

4.2. Calabi-Yau manifolds and mirror symmetry 289

4.3. Mathematics inspired by mirror symmetry 291

5. Invariants of Calabi-Yau manifolds 291

5.1. Gromov-Witten invariants 291

5.2. Counting formulas 292

5.3. Proofs of counting formulas for Calabi-Yau threefolds 293

5.4. Integrability of mirror map and arithmetic applications 293

5.5. Donaldson-Thomas invariants 294

5.6. Stable bundles and sheaves 296

5.7. Yau-Zaslow formula for K3 surfaces 296

5.8. Chern-Simons knot invariants, open strings and string dualities 
6. Homological mirror symmetry 299

7. SYZ geometric interpretation of mirror symmetry 300

7.1. Special Lagrangian submanifolds in Calabi-Yau manifolds

7.2. The SYZ conjecture - SYZ transformation 301

7.3. Special Lagrangian geometry 301

7.4. Special Lagrangian fibrations 302

7.5. The SYZ transformation 303

7.6. The SYZ conjecture and tropical geometry 303

8. Geometries related to Calabi-Yau manifolds 304

8.1. Non-Kähler Calabi-Yau manifolds 304

8.2. Symplectic Calabi-Yau manifolds 305

References 306

\section{Introduction}

Calabi-Yau manifolds are compact, complex Kähler manifolds that have trivial first Chern classes (over $\mathbb{R}$ ). In most cases, we assume that they have finite fundamental groups. By the conjecture of Calabi [45] proved by Yau $[\mathbf{2 9 3}, \mathbf{2 9 5}]$, there exists on every Calabi-Yau manifold a Kähler metric with vanishing Ricci curvature.

Currently, research on Calabi-Yau manifolds is a central focus in both mathematics and mathematical physics. It is partially propelled by the prominent role the Calabi-Yau threefolds play in superstring theories. While many beautiful properties of Calabi-Yau manifolds have been discovered, more questions have been raised and probed. The landscape of various constructions, theories, conjectures, and above all the fast pace progress in this subject, have made the research of Calabi-Yau manifolds an extremely active research field both in mathematics and in mathematical physics.

Note: In writing an overview of such a broad subject area, the need to be inclusive was recognized and many experts were consulted. But unfortunately, in the citing of original references and the topics covered, omissions inevitably always occur, and for this, sincere apology is offered.

\section{General constructions of complete Ricci-flat metrics in Kähler geometry}

2.1. The Ricci tensor of Calabi-Yau manifolds. A complex manifold is a topological space covered by complex coordinate charts such that the transition between overlapping charts are holomorphic; a Hermitian metric on a complex manifold is a smooth assignment of Hermitian inner product structures on the holomorphic tangent spaces of the manifold; a Hermitian metric is called a Kähler metric if near every point the Hermitian metric is approximated by a flat metric up to second order. In a holomorphic coordinate chart with coordinate variables $\left(z_{1}, \ldots, z_{n}\right)$, a Hermitian metric has its 
associated Hermitian form

$$
\omega=\frac{\sqrt{-1}}{2} \sum g_{i j} d z^{i} \wedge d \bar{z}^{j}
$$

A Hermitian metric is Kähler if its Hermitian form is closed. In such a case, we call the Hermitian form the Kähler form of the Kähler metric.

Given any Kähler metric, one defines its full curvature tensor by certain expressions of covariant derivatives of the metric; the Ricci curvature is a partial contraction of the full curvature tensor. The indices of the Ricci curvature is identical to that of the Kähler form. In local coordinates,

$$
R i c=\sqrt{-1} \sum R_{i \bar{j}} d z_{i} \wedge d \bar{z}_{j}
$$

We call a Kähler metric Ricci-flat if its Ricci tensor vanishes identically.

2.2. The Calabi conjecture. According to a well-known theorem of Chern, the Ricci form divided by $2 \pi$ is a $(1,1)$-form that represents the first Chern class of a compact complex manifold. Rooted in his attempt to find canonical Kähler metrics for a Kähler manifold, in 1954, E. Calabi [45] proposed his celebrated conjecture.

Conjecture. To every closed $(1,1)$-form $\frac{1}{2 \pi} C_{1}(X)$ representing the first Chern class $c_{1}(X)$ of a Kähler manifold $X$, there is a unique Kähler metric in the same Kähler class whose Ricci tensor (form) is the closed $(1,1)$ form $C_{1}(X)$.

In case the complex manifold has vanishing first Chern class, the zero form represents the first Chern class of the manifold. The Calabi conjecture implies the existence of a unique Ricci-flat Kähler metric in every Kähler class.

Early on, Calabi realized that his conjecture can be reduced to a complex Monge-Ampère equation.

2.3. Yau's theorem. By the late 1960s, many were doubtful of the Calabi conjecture. Some attempted to use a reduction theorem of CheegerGromoll [64] (in 1971) to construct counterexamples to the Conjecture. Using the reduction theorem and assuming the conjecture, Yau announced the following splitting theorem in his 1973 lecture at the Stanford geometry conference: Every compact Kähler manifold with non-negative Ricci curvature can be covered by a metric product of a torus and a simply connected manifold with a Ricci-flat Kähler metric. He then used this theorem to produce a "counterexample" to the conjecture. The "counterexample" was soon discovered to be flawed; Yau withdrew his Stanford lecture. (The flaw was due to the mistaken assumption that manifolds with numerically nonnegative anti-canonical divisor admits a first Chern form which is pointwise non-negative.) 
In 1976, Yau $[\mathbf{2 9 3 , 2 9 5}$ proved the Calabi conjecture by solving the complex Monge-Ampère equation for a real valued function $\phi$

$$
\operatorname{det}\left(g_{i \bar{j}}+\frac{\partial^{2} \phi}{\partial z^{i} \partial \bar{z}^{j}}\right)=e^{f} \operatorname{det}\left(g_{i \bar{j}}\right),
$$

where $e^{f}$ is any average 1 smooth function and $g_{i \bar{j}}+\partial_{i} \partial_{\bar{j}} \phi$ is required to be positive definite. The solution $\phi$ of the above equation ensures that the new Kähler metric

$$
\omega+\sqrt{-1} \partial \bar{\partial} \phi
$$

can attain Ricci (curvature) form in any form referred to in the Calabi conjecture.

2.4. Calabi-Yau manifolds and Calabi-Yau metrics. The first application to Yau's proof of Calabi conjecture is the existence of Ricciflat Kähler metric on every compact complex Kähler manifold with trivial canonical class. (Trivial canonical class is equivalent to the existence of a nowhere vanishing holomorphic volume form, which is equivalent to that the top wedge power of the holomorphic cotangent bundle is the trivial line bundle.) The converse is also true: any Ricci-flat simply connected Kähler manifold has trivial canonical line class. This proves the existence and provides a criterion for Kähler Calabi-Yau manifolds.

By convention, Calabi-Yau manifolds exclude those with infinite fundamental groups. The Ricci-flat metrics on Calabi-Yau manifolds are called Calabi-Yau metrics.

The existence of Calabi-Yau metrics has other important consequences. In his paper $[\mathbf{2 9 3}, \mathbf{2 9 5}]$, Yau demonstrated that for a Calabi-Yau manifolds $(X, \omega)$, using the Chern-Weil form representing the second Chern form $C_{2}(X)$ in terms of the curvature tensor $R m$ of a Calabi-Yau Kähler metric of $X$, one gets

$$
\int_{X} C_{2}(X) \wedge \omega^{n-2}=C \int_{X}|R m|^{2} \operatorname{vol} \geq 0
$$

for some positive constant $C$. Thus the Chern number $c_{2}(C) \cap[\omega]^{n-2}$ is nonnegative. Moreover, when it is zero, we have $R m=0$, and therefore $X$ is covered by the Euclidean space $\mathbb{C}^{n}$.

Another application is the reduction of holonomy groups of Calabi-Yau manifolds. One important consequence of a Calabi-Yau metric is that the parallel transports along contractible closed loops preserve the metric and the holomorphic volume form. This implies that the restricted holonomy group of a Calabi-Yau manifold is a subgroup of $S U(n)$, the group of special unitary transformations.

Sometimes, this group can be strictly smaller than $S U(n)$. Following the Bochner technique on Calabi-Yau manifolds, every holomorphic $(p, 0)$-form is parallel. Such a form then reduces the holonomy group from $S U(n)$ to a smaller subgroup. Thus if the holonomy group of $X$ is the full $S U(n)$, then 
the Dolbeault cohomology group $H^{p, 0}(X)=0$ for $1 \leq p \leq n-1$ and $H^{n, 0}(X)$ is one dimensional, spanned by the holomorphic volume form $\Omega$ of $X$.

The statement of the structure theorem was known to many people, including the announcement made by Yau in 1973. It was also announced by Kobayashi, by Michelsohn [218], and appeared in a subsequent survey paper of Beauville [19]. It states that any compact, complex Kähler manifold with trivial canonical class has a cover that is a metric product of a complex Euclidean space with copies of manifolds with holonomy groups $S U(m)$ and copies of manifolds with holonomy groups $S p(m / 2)$; here $m$ 's are the dimensions of the corresponding manifolds.

The proof is based on the above-mentioned splitting theorem of CheegerGromoll and also an argument of Calabi who drew some consequences on the first Betti number; this construction of Calabi is referred to as the Calabi construction in the sixties.

2.5. Examples of compact Calabi-Yau manifolds. By Yau's solution to the Calabi conjecture, finding (non-hyperkähler) Calabi-Yau manifolds is equivalent to finding smooth projective varieties of trivial canonical class.

The first example of a Calabi-Yau threefold is the smooth quintic in the complex projective space $\mathbb{P}^{4}$. Due to a condition imposed by superstrings theories, Calabi-Yau threefolds having Euler characteristic $\chi= \pm 6$ and non-trivial fundamental group play a special role. Such examples were first discovered and announced by Yau in a lecture given at the 1985 Argonne conference $[\mathbf{2 9 6}]$ as the $\mathbb{Z}_{3}$ quotient of an intersection of two cubics and a hypersurface of bi-degree $(1,1)$ in the product $\mathbb{P}^{3} \times \mathbb{P}^{3}$. More examples were found later by Tian and Yau; due to an observation of Greene and Kirklin [115], these examples are deformation-equivalent to the one found by Yau. A systematic search for Calabi-Yau threefolds with $\chi= \pm 6$ turned up no essentially new example among complete intersections in toric varieties [59].

After Yau's examples of complete intersection Calabi-Yau threefolds in product of projective spaces, various groups, notably the group in University of Texas, employed computer algorithm to search for new examples $[\mathbf{1 4 7}, \mathbf{1 1 7}, \mathbf{4 8}]$. Soon after, about 8000 constructions, with 256 distinct Hodge diamonds [120] were found. This pool grew by about 3 orders of magnitude by embedding in products of weighted complex projective spaces [60]. Such threefolds typically have finite quotient singularities inherited from the weighted projective spaces, and a minimal blow-up following Roan and Yau's 1987 construction [245] is understood to provide smooth models. Roan and Yau also proposed to use toric method to construct more examples. This was carried out by Batyrev and Borisov for complete intersections in toric varieties $[\mathbf{1 4}]$.

Yau conjectured that there are finitely many topological types of CalabiYau manifolds in each dimension. This conjecture is still open. By a rough count, by 2002, over 473 million toric embeddings of Calabi-Yau threefolds 
were constructed, with over 30,000 distinct Hodge diamonds [169, 170]. Based on Wall's theorem [281] one sees that complete intersections of hypersurfaces in products of projective spaces produce at least 2590 distinct diffeomorphism classes [57]. It is worth noticing that so far all Calabi-Yau threefolds can be constructed as deformations or small resolutions of complete intersections of toric varieties. The non-trivial check of this for CalabiYau complete intersections in Grassmannians and flag varieties was done by Batyrev, Ciocan-Fontanine, Kim and van Straten $[\mathbf{1 5}, \mathbf{1 6}]$.

2.6. Noncompact Calabi-Yau manifolds. Immediately after his proof of the Calabi conjecture, Yau generalized the construction of CalabiYau manifolds to non-compact Kähler manifolds. He presented this result in his plenary lecture in the 1978 Helsinki International Congress of Mathematics.

The construction is that in the complement $M \backslash S$ of a compact Kähler manifold $M$ with an effective anticanonical divisor $S$ removed, suppose the first Chern class of $M$ is either positive or trivial in a neighborhood of $S$, then there is a complete Ricci-flat metric on $M \backslash S$ if $S$ is connected and geometrically stable.

In case $S$ is nonsingular and connected and assuming $S$ admits a Kähler Einstein metric with either positive or zero scalar curvature, the detail of the generalization was presented in a joint paper of Tian-Yau $[\mathbf{2 7 1}, \mathbf{2 7 2}]$. Around the same time, Bando-Kobayashi $[\mathbf{1 1}, \mathbf{1 2}]$ worked out some more restrictive cases. The condition that $S$ should be geometrically stable was added on after the Helsinki Congress. When $S$ is singular, the definition need to be clarified.

If the complement $M \backslash S$ admits a complete Ricci-flat metric, $S$ has to be connected unless the complement is a product of the complex line with other manifolds. Based on his result on the volume growth of complete manifolds with non-negative Ricci curvature and compactification of complete Kähler manifolds, Yau conjectured that his construction gives all examples of noncompact complete Kähler Ricci-flat metrics with connected end. There are counterexample to this conjecture, Taub-NUT metric is one such example was pointed out by Anderson-Kronheimer-LeBrun [3]. The conjecture remains open assuming the manifolds are of finite topological type.

Explicit Calabi-Yau metrics have been constructed in many cases when symmetries are present. First there is the explicit Eguchi-Hanson metric [89] on the cotangent bundle $T^{*} S^{2}$ of the two sphere. Viewing this as the canonical line bundle over $\mathbb{P}^{1}$, Calabi $[46]$ constructed a complete Calabi-Yau metric on the total space $X=K_{B}$ of (a fraction of) the canonical line bundle of a positive Kähler-Einstein manifold $B$. Later, Futaki [104] generalized to that $B$ is any toric Fano manifold.

Candelas and de la Ossa [49] constructed a one-parameter family of explicit Calabi-Yau metrics on the cotangent bundle $T^{*} S^{3}$ of the three sphere, by reducing the Ricci-flat equation to an ODE. The parameter of this 
family corresponds to the size of $S^{3}$. This was generalized to all dimensions by Stenzel $[\mathbf{2 6 3}]$.

Candelas and de la Ossa [49] also constructed a one-parameter family of explicit Calabi-Yau metrics on the total space of the vector bundle $X=$ $\mathcal{O}(-1) \oplus \mathcal{O}(-1)$ over $\mathbb{P}^{1}$. As the size of $\mathbb{P}^{1}$ shrinks to zero, the total space $X$ degenerates to a cone threefold $X_{0}$ with rational double point singularity

$$
X_{0}=\left\{z_{0}^{2}+z_{1}^{2}+z_{2}^{2}+z_{3}^{2}=0\right\} \subset \mathbb{C}^{4} .
$$

This singular space has two different small resolutions $X_{+}$and $X_{-}$, both have total space $\mathcal{O}(-1) \oplus \mathcal{O}(-1)$. The pair $\left(X_{+}, X_{-}\right)$is a local model of a simple flop, a birational change of threefolds.

If one varies the quadratic equation $z_{0}^{2}+z_{1}^{2}+z_{2}^{2}+z_{3}^{2}=t$ by adding a small nonzero $t$, then it defines a smooth hypersurface $X_{t}$ in $\mathbb{C}^{4}$ that is diffeomorphic to $T^{*} S^{3}$. The change from $X_{ \pm}$to $X_{t}$ is called an extremal transition; it is the basic building block for topological changes of Calabi-Yau threefolds.

2.7. Calabi-Yau cones: Sasaki-Einstein manifolds. An important class of non-compact and possibly incomplete Calabi-Yau manifolds are Calabi-Yau cones. The metric cone over a compact Riemannian manifold $(S, g)$ is defined to be $\left(C(S)=\mathbb{R}_{+} \times S, \bar{g}=d r^{2}+r^{2} g\right)$, where $r>0$ is a coordinate on $\mathbb{R}_{+}$. If the dimension of this cone is $2 n$ and the (restricted) holonomy group of $(C(S), \bar{g})$ is contained in $S U(n)$, then the manifold $(S, g)$ is called Sasaki-Einstein. In particular, since the cone is Ricci-flat it follows that $(S, g)$ is a $(2 n-1)$-dimensional Einstein manifold of positive Ricci curvature, $\mathrm{Ric}_{g}=2(n-1) \mathrm{g}$. In fact, many of the complete non-compact CalabiYau manifolds referred to above are asymptotic to such a cone, meaning that they are modelled at infinity by the large $r$ (complete) end of the cone. Sasaki-Einstein manifolds in low dimensions are also important in string theory, and in particular in the AdS/CFT correspondence. For example, the latter conjectures that to every Sasaki-Einstein 5-manifold there is an associated superconformal field theory on $\mathbb{R}^{4}$. Much work has gone into understanding this correspondence, and the relationship between Sasaki-Einstein geometry and superconformal field theory.

The definition above is easily generalized: if the metric cone has (restricted) holonomy contained in $U(n)$, so that the cone is Kähler, the manifold $(S, g)$ is said to be a Sasakian manifold [252]. These manifolds should be viewed as odd-dimensional analogs of Kähler manifolds. A Sasakian manifold inherits a strictly pseudo-convex hypersurface-type CR structure from the complex structure of the cone. Sasakian manifolds are also equipped with a unit norm Killing vector field $\xi$, called the Reeb vector field, defined as the restriction of $J(r \partial / \partial r)$ to $\{r=1\} \cong S \subset C(S)$, where $J$ is the complex structure tensor of the Kähler cone. The dual one-form $\eta(X)=g(\xi, X)$ is a contact form on $S$. The flow of $\xi$ defines a one-dimensional foliation of $S$, and it turns out that the transverse leaf space is Kähler. Indeed, $(S, g)$ is SasakiEinstein if and only if this transverse Kähler structure, with transverse 
metric $g^{T}$, is Kähler-Einstein with positive Ricci curvature, $\operatorname{Ric}_{g^{T}}=2 n g^{T}$. Sasakian manifolds may be classified according to the global properties of this foliation. If the orbits of $\xi$ all close, thus defining a locally free circle action on $S$, the Sasakian manifold is said to be quasi-regular, and the leaf space is naturally a Kähler orbifold. In the special case that the circle action is free, the Sasakian manifold is said to be regular, and the leaf space is a Kähler manifold. If there is a non-closed orbit of $\xi$ the Sasakian manifold is said to be irregular.

The simplest example of a Sasaki-Einstein manifold is the round sphere, viewed as the unit sphere in $\mathbb{C}^{n}$ equipped with its flat Kähler metric. This is regular, with the Kähler-Einstein leaf space being $\mathbb{C P}^{n-1}$ equipped with its Fubini-Study metric. The study of regular Sasaki-Einstein manifolds is in fact essentially equivalent to the study of positive (Fano) Kähler-Einstein manifolds. Boyer-Galicki $[\mathbf{3 3}, \mathbf{3 4}]$ and their collaborators have constructed large classes of quasi-regular Sasaki-Einstein manifolds by constructing appropriate Kähler-Einstein orbifold leaf spaces. These are typically realized as weighted projective varieties, and the continuity method is used to prove existence, see $[\mathbf{2 8 3}, \mathbf{8 3}]$ for the existence of Kähler-Einstein metrics on toric varieties. Boyer-Galicki-Kollár [35] have also shown the existence of numerous Sasaki-Einstein metrics on standard and exotic spheres using the Kähler-Einstein metrics on certain types of Fano orbifolds constructed by Demailly-Kollár [72].

The first examples of irregular Sasaki-Einstein manifolds were constructed by Gauntlett-Martelli-Sparks-Waldram [108]; these authors constructed infinitely many explicit quasi-regular and irregular Sasaki-Einstein metrics on $S^{2} \times S^{3}$. Recently, Futaki-Ono-Wang [105] have proven the existence of toric Sasaki-Einstein metrics, following earlier work of Martelli-Sparks-Yau [214]. In this case the Kähler cone is the smooth part of an affine toric variety. Finally, Gauntlett-Martelli-Sparks-Yau [108] have described some simple obstructions to the existence of Sasaki-Einstein metrics, which also give new obstructions to the existence of Kähler-Einstein metrics on Fano orbifolds.

2.8. The balanced condition on Calabi-Yau metrics. After observing that the tangent bundles of Calabi-Yau manifolds are stable with respect to any polarization, Yau conjectured that the Calabi-Yau manifolds are also stable in the sense of geometric invariant theory. This was first openly discussed in the problem session in the UCLA geometry conference in 1990, where he proposed to approximate the Ricci-flat metric of a CalabiYau manifold $X$ by the induced metric from embedding $X$ into complex projective space by powers of an ample line bundle, and suggested that the action of the projective linear group on the embedding would link the stability of the manifold with the existence of the Ricci-flat metric.

Yau [297] initiated the program of approximating Calabi-Yau metrics (and more generally KE-metrics) by embeddings. Under his guidance, Tian 
[270] wrote his thesis on the $C^{2}$ convergence of the pullback Fubini-Study metric via projective embeddings. (The $C^{\infty}$ convergence was proved in Ruan's Harvard thesis [246]. See also [70].) The refined structure of this embedding was investigated by Catlin [61], Zelditch [300] and Lu [208]. For the problem of finding a canonical position in embedding a Calabi-Yau manifold in projective space, the balanced condition introduced by BourguignonLi-Yau in [32] played a crucial role. (The idea of Bourguignon-Li-Yau was based on the concept of the conformal area introduced by Li-Yau [191] in 1982.) Following their idea, Luo [210] in his MIT thesis (under the guidance of Yau) generalized the notion of balanced embeddings to all projective manifolds and related the concept to questions of geometric stability. The relation between geometric stability and the balanced condition was also studied by Zhang [301].

This program of Yau was carried out by Donaldson $[\mathbf{8 0}, \mathbf{8 1}]$ in more precise manner. Donaldson showed that for the sequence of balanced embeddings of a Calabi-Yau manifold into projective spaces via increasing powers of an ample line bundle, the sequence of normalized induced metrics converges to the Ricci-flat metric of $X[\mathbf{8 0}]$. (See $[\mathbf{2 0 2}]$ for some clarifications.) Based on the balanced embeddings, he then developed an algorithm to numerically approximate the Ricci-flat metrics of K3 surfaces [81]. This algorithm was generalized by Douglas-Karp-Lukic-Reinbacher [87], and subsequently Braun-Brelidze-Douglas-Ovrut $[\mathbf{3 7}, \mathbf{3 8}]$ to approximate the Ricciflat metrics on various projective Calabi-Yau threefolds.

The work of Donaldson also showed that every Calabi-Yau manifold is asymptotically Chow stable, proving partially Yau's conjecture on the stability of Kähler Einstein manifolds [80].

\section{Moduli and arithmetic of Calabi-Yau manifolds}

3.1. Moduli of K3 surfaces. Two dimensional Calabi-Yau manifolds are K3 surfaces. Moduli of K3 surfaces are classically known to be smooth. It has a modular description based on the Hodge structures on the $K 3$ surfaces.

On any $\mathrm{K} 3$ surface $X$, the middle cohomology group $H^{2}(X, \mathbb{Z})$ is a free Abelian group of rank 22 and coupled with the (intersection) quadratic form $\langle\cdot, \cdot\rangle$ the lattice $\left(H^{2}(X, \mathbb{Z})\langle\cdot, \cdot\rangle\right)$ is isometric to the lattice

$$
L:=\left(\mathbb{Z}^{\oplus 22},-E_{8} \oplus-E_{8} \oplus U \oplus U \oplus U\right),
$$

where $E_{8}$ is the Cartan matrix of the corresponding root system and $U$ is the rank two hyperbolic matrix.

The holomorphic 2-form $\Omega$ on $X$, which is unique up to scalars, spans a ray in $H^{2}(X, \mathbb{Z}) \otimes_{\mathbb{Z}} \mathbb{C}$ and satisfies the well-known Riemann bilinear relation

$$
\langle\Omega, \Omega\rangle=0, \text { and }\langle\Omega, \bar{\Omega}\rangle>0 \text {. }
$$


Thus after fixing a marking of $X$ that is an isomorphism $\left(H^{2}(X, \mathbb{Z})\langle\cdot, \cdot\rangle\right) \cong$ $L$, the period $[\Omega]$ lies in

$$
\mathcal{D}=\left\{v \in \mathbb{P} L_{\mathbb{C}} \mid\langle v, v\rangle=0,\langle v, \bar{v}\rangle>0\right\} .
$$

This is the period domain for K3 surfaces, and the assignment $X \mapsto[\Omega]$ for marked Kähler K3 surface $X$ is called the period map.

The Torelli problem is on how the element $[\Omega] \in \mathcal{D}$ determines the complex structure of a marked Kähler K3 surface. The global Torelli theorem for algebraic surfaces was proved by Pjateckii-Šapiro and Šafarevič $[\mathbf{2 4 1}]$; the Torelli theorem for Kähler $K 3$ surfaces, proved by Burns-Rapoport [43] and Looijenga-Peters [207], states that the loci of the holomorphic two-form $[\Omega]$ in $\mathcal{D}$ uniquely determines the marked K3 surface $X$ up to an isomorphism of $L$ generated by Picard-Lefschetz transformation.

The surjectivity of the period map was proved Kulikov [171] followed by Persson-Pinkham [238]. The approach based on the Calabi-Yau metric was pioneered by Todorov $[\mathbf{2 7 3}]$ and completed by Siu $[\mathbf{2 5 7}]$ and Looijenga [206]. The proof relies heavily on Yau's solution of the Calabi conjecture. Later, based on Yau's solution of the Calabi conjecture, the main lemma of Burns-Rapoport [43], and with the surjectivity of the period map of Kähler $K 3$ surfaces, Todorov and Siu [258] proved that every $K 3$ surface is Kähler.

3.2. Moduli of high dimensional Calabi-Yau manifolds. The existence of the moduli of polarized Calabi-Yau manifolds was settled by the work of Viehweg [279]. The next question is the regularity of the moduli space. The first theorem was due to Bogomolov [30] who proved that the universal deformation space of a compact Kähler-Hamiltonian manifold is unobstructed. In one of his unpublished manuscript, he also claimed that the same is true for any projective manifold with trivial first Chern class.

Todorov [274] and Tian [269] each confirmed this claim by proving that every Calabi-Yau manifold has unobstructed deformations. Both proofs used essentially the Calabi-Yau metric of the manifold to derive a differentialgeometric computational equality that allows them to solve the Kuranishi equation in analytic deformation theory. This theorem is now referred to as the Bogomolov-Tian-Todorov unobstructedness theorem.

Ran $[\mathbf{2 4 3}]$ and Kawamata $[\mathbf{1 5 6}, \mathbf{1 5 7}]$ gave new proofs of this unobstructedness result, based on the notion of $T^{1}$-lifting property. Their method was later applied to non-Kählerian Calabi-Yau manifolds and to some singular projective Calabi-Yau varieties.

On the moduli space, there is a natural Kähler metric obtained from the variation of the Ricci-flat metric called the Weil-Petersson metric. The volume of the moduli space of polarized Calabi-Yau manifolds with respect to this metric was proved by Lu-Sun $[\mathbf{2 0 9}]$ and Todorov $[\mathbf{2 7 5}]$ to be finite. This follows from finding a suitable metric that bounds the Weil-Petersson metric from above and satisfies the conditions of the Scwharz lemma [294], 
which implies generally that for any Hermitian metric defined on a quasiprojective manifold whose Ricci curvature has a strongly negative upper bound, the total volume is finite.

The study of the moduli space of complex structures on a Calabi-Yau threefold led Hitchin to study invariant functionals on differential forms [134]. This approach is also useful when studying the associated flow equations that describes the geometry in terms of an evolving hypersurface. This approach has also led Hitchin [135] to develop the geometry based on open orbits of $G L(n, \mathbb{R})$ on $k$-forms, especially when $k=3[\mathbf{1 3 4}]$.

As observed later, many examples of pairs of topologically distinct Calabi-Yau threefolds can be connected by flops or by small contractions followed by smoothing. For instance, Kawamata [158] has recently proved that any two birational smooth Calabi-Yau manifolds can be connected by a sequence of flops. (Kawamata's general result is valid in any complex dimension. The proof for threefolds was given earlier by Kollár [162] and fourfolds by Burns-Hu-Luo [42].) One might speculate that the collection of all Calabi-Yau threefolds can be connected by such process. Reid raised this as his fantasy [244].

For high dimensional Calabi-Yau manifolds, a major question is the Torelli problem. For hyperkähler manifolds, Huybrechts $[\mathbf{1 4 9}]$ proved that the period map from the moduli space of marked hyperkähler manifolds to the period domain is surjective. The case of general Calabi-Yau manifolds has been recently studied by Liu-Sun-Todorov-Yau.

3.3. The modularity of Calabi-Yau threefolds over $\mathbb{Q}$. In search of Calabi-Yau manifolds that distinguish themselves from the rest, their modularity become the focus of some researchers. This is interesting from the perspective of arithmetic geometry.

For a $d$-dimensional projective Calabi-Yau manifolds $X$ defined over $\mathbb{Q}$, it is said to be modular if the $L$-function of the Galois representation on the middle $\ell$-adic étale cohomology group $H_{e t}^{d}\left(X_{\overline{\mathbb{Q}}}, \mathbb{Q}_{\ell}\right)$ is equal to the product of $L$-functions of modular forms up to factors associated to bad primes. Part of Langlands philosophy is the conjecture that all motives, in particular our $X$, are modular. When $d$ is even, $H_{e t}^{d}\left(X_{\overline{\mathbb{Q}}}, \mathbb{Q}_{\ell}\right)$ contains $d / 2$-dimensional algebraic cycles, and the interesting part is the modularity of the sub-representation on the orthogonal complement of the images of algebraic cycles.

Calabi-Yau varieties of dimension 1 are elliptic curves. The modularity of elliptic curves over $\mathbb{Q}$ has been established by Wiles [284], and TaylorWiles $[\mathbf{2 6 6}]$. They proved that the two-dimensional Galois representation associated to an elliptic curve over $\mathbb{Q}$ does come from a weight $2=d+1$ modular form.

Dimension 2 Calabi-Yau varieties are K3 surfaces. For a K3 surface $X$ defined over $\mathbb{Q}, H_{\text {et }}^{2}\left(X_{\overline{\mathbb{Q}}}, \mathbb{Q}_{\ell}\right)$, which has dimension 22 , factors into a direct 
sum $(N S(X) \oplus T(X)) \otimes \mathbb{Q}_{\ell}$ of the Néron-Severi group of algebraic cycles $N S(X)$ and the group of transcendental cycles $T(X)$.

There are partial result for small rank $T(X)$. The lattice $N S(X)$ has rank at most 20; when it is $20, X$ is called a singular $\mathrm{K} 3$ surface. (The term "attractive" $\mathrm{K} 3$ is sometimes also used in physics [223].) In this case, $T(X)$ has rank 2 and defines a two-dimensional Galois sub-representation and the associated $L$-function $L(T(X), s)$. The modularity of $L(T(X), s)$ has been established by Livné $[\mathbf{2 0 4}]$ that $L(T(X), s)$ does come from a weight $3=d+1$ modular form of CM type. When $T(X)$ has rank 3 , its modularity follows from the modularity of elliptic curves, because $T(X)$ is endowed with an orthogonal pairing, so that it is essentially the symmetric square of a $G L(2)$ representation.

For Calabi-Yau threefolds defined over $\mathbb{Q}$, much is known for the rigid case, where there is no complex structure deformation. More specifically, we say that a Calabi-Yau threefold $X$ is rigid if the $H_{e t}^{3}\left(X_{\overline{\mathbb{Q}}}, \mathbb{Q}_{\ell}\right)$ has dimension 2. In this case, there is a two-dimensional Galois representation associated to $X$; the modularity has been established, under some mild conditions, that $L(X, s)$ is determined by some weight $4=d+1$ modular forms.

It is worth noticing that currently more than 50 modular rigid CalabiYau threefolds over $\mathbb{Q}$ have been constructed, and expanding.

The modularity question for a non-rigid Calabi-Yau threefold $X$ over $\mathbb{Q}$ poses more serious challenge as the dimension of $H_{e t}^{3}\left(X_{\overline{\mathbb{Q}}}, \mathbb{Q}_{\ell}\right)$ gets larger. Much less is known.

An attractor flow equation on the complex structure moduli space of Calabi-Yau threefolds was found by Ferrara-Kallosh-Strominger [93] in their study of BPS black holes solutions in string theory. Moore [223] has shown that Calabi-Yau manifolds with complex structure located at an attractor fixed point on the moduli space exhibit interesting arithmetic properties.

\section{Calabi-Yau manifolds in physics}

Calabi-Yau manifolds admit Kähler metrics with vanishing Ricci curvatures. They are solutions of the Einstein field equation with no matter. The theory of motions of circles inside of a Calabi-Yau manifold provide a model of a conformal field theory. (It is called a $\sigma$-model in physics.) Because of this, Calabi-Yau manifolds are pivotal in superstring theory.

4.1. Calabi-Yau manifolds in string theory. Superstring theory is a unified theory for all the forces of nature including quantum gravity. In superstring theory, the fundamental building block is an extended object, namely a string, whose vibrations would give rise to the particles encountered in nature. The constraints for the consistency of such a theory are extremely stringent. They require in particular that the theory takes place in a 10dimensional space-time. To make contact with our 4-dimensional world, it is expected that the 10-dimensional space-time of string theory is locally 
the product $M^{4} \times X$ of a 4 -dimensional Minkowski space $M^{3,1}$ with a 6dimensional space $X$. The 6 -dimensional space $X$ would be tiny, which would explain why it has not been detected so far at the existing experimental energy levels. Each choice of the internal space $X$ leads to a different effective theory on the 4-dimensional Minkowski space $M^{3,1}$, which should be the theory describing our world.

It has long been argued that, in order to solve certain classic problems of unified gauge theories such as the gauge hierarchy problem, the 4-dimensional effective theory should admit an $N=1$ supersymmetry. In a fundamental paper, Candelas-Horowitz-Strominger-Witten [58] analyzed what the constraint of that $N=1$ supersymmetry would mean for the geometry of the internal space $X$. They found that, for the most basic product models with $N=1$ supersymmetry, the space $X$ must be a Calabi-Yau manifold of complex dimension 3. Shortly afterwards, Strominger [264] considered slightly more general models, allowing warped products. For these models, the $N=1$ supersymmetry constraint results in a modification of the Ricci-flat equation of the earlier model.

4.2. Calabi-Yau manifolds and mirror symmetry. Around 19871988, physicists including Dixon [76], and Lerche, Vafa, and Warner [181] observed that in mapping an abstract $N=2$ superconformal field theory to a possible geometrical realization as a Calabi-Yau sigma model, an ambiguity arose. A superconformal field theory has two natural rings (called (c,c) and (a,c) rings)) as does a Calabi-Yau sigma model (the Dolbeault cohomology naturally splits into even and odd dimensional forms). The question which came to light was which of the two possible pairings of the conformal field theory and geometrical rings is induced by the map between the superconformal theory and the Calabi-Yau sigma model. Lerche, Vafa, and Warner conjectured that maybe both pairings are realized because, they suggested, Calabi-Yau threefolds come in pairs in which the even and odd cohomologies are interchanged. (To be precise, the interchange is between the Dolbeault cohomology $H^{(p, q)}$ with $H^{(3-p, q)}$ for Calabi-Yau threefolds.) At the time, the evidence in support of this conjecture was thin. Some suggested that a less radical solution to the observed ambiguity might be to keep the base Calabi-Yau manifold fixed and merely consider completing the geometrical model in two ways: by including its tangent bundle or its co-tangent bundle.

Nevertheless, in 1989, Greene and Plesser [116], using the methods of conformal field theory as applied to Calabi-Yau sigma models realized as twisted products of $N=2$ minimal models, were able to establish that certain pairs of Calabi-Yau manifolds come in pairs in which their Hodge diamonds are mirror reflections (through a diagonal) of one another. Moreover, Greene and Plesser were able to establish that these pairs of CalabiYau manifolds, even though topologically distinct, when used as the basis for Calabi-Yau sigma models, give the same physical string theory. They named such pairs of Calabi-Yau manifolds mirror manifolds. The existence 
of such pairs of Calabi-Yau manifolds with specified properties are known to mathematicians as the Mirror Symmetry conjecture.

Of the few hundred mirror manifold pairs which Greene and Plesser's approach explicitly generated, the most famous example is the Fermat quintic $X$ in $\mathbb{C P}^{4}$ defined by the vanishing of

$$
f\left(z_{0}, z_{1}, \ldots, z_{4}\right)=z_{0}^{5}+z_{1}^{5}+\cdots+z_{4}^{5}+\psi\left(z_{0} z_{1} \cdots z_{4}\right)
$$

with its mirror being a crepant resolution of $X /\left(\mathbb{Z}_{5}\right)^{3}$, known as the mirror quintic.

Beyond constructing such pairs of mirror manifolds, Greene and Plesser noted that one implication of having a mirror pair yielding identical physical models is the existence of a highly nontrivial identity involving the so-called Yukawa couplings of each-quantities determined by the (quantum deformations of the) even cohomology ring of one manifold and the odd cohomology ring of its mirror.

A short time later, Candelas, de la Ossa, Green and Parkes (CDGP) [52], studied the example of the mirror quintic pair by undertaking a detailed examination of the variation of Hodge structures of the mirror quintic. This work, interpreted mathematically by Morrison [221] and AspinwallMorrison [6], produced a beautiful solution to a long-standing problem in enumerative geometry - "counting" rational curves on a general quintic. It is important to note that this work relied on another conjecture - the mirror map conjecture - purporting to give the explicit map between the moduli spaces of this pair of Calabi-Yau mirror manifolds. The foundational discovery of Greene and Plesser, and of Candelas-de la Ossa-Green-Parkes, helped set in motion one of the most spectacular developments in modern mathematics.

A far-reaching generalization of the (genus zero) variation of Hodge structure - the so-called Kodaira-Spencer theory of gravity of Bershadsky, Cecotti, Ooguri and Vafa (BCOV) [27] - later led to conjectural counting formulas for GW invariants of all genera for many Calabi-Yaus.

BCOV generalizes the variation of Hodge structure incorporating a natural hermitian structure which comes from the special Kähler geometry on the moduli space of Calabi-Yau manifolds. The generalized theory of Hodge structure is regarded as a special case of " $t-t^{*}$ geometry" of two dimensional $N=2$ supersymmetric QFT due to Cecotti-Vafa [62]. At genus zero, $t-t^{*}$ geometry includes the successful associative relation, called WittenDijkgraaf-Verlinde-Verlinde $[\mathbf{2 8 6}, \mathbf{7 5}]$ (WDVV) equation, in quantum cohomology of projective manifolds. In 1993, BCOV [26] conjectured that one point function on a torus in the $t-t^{*}$ geometry of a Calabi-Yau manifold provides a non-trivial extension of the CDGP counting formula to genus one, called BCOV genus-one formula. Soon after, BCOV [27] introduced a certain recursion formula for higher genus $(g \geq 2) \mathrm{GW}$ invariants, which is called the holomorphic anomaly equation. This recursion formula due to BCOV is still under extensive study for its mathematical ground. 
A physical proof of mirror symmetry has been given by Hori-Vafa [137]. They demonstrated the equivalence at the level of two-dimensional gauged linear sigma model [289] which in the low-energy limit leads to the conformal field theory with Calabi-Yau manifold target space. (See also MorrisonPlesser [222] for an earlier attempt along the same lines.)

4.3. Mathematics inspired by mirror symmetry. One area inspired by the mirror symmetry conjecture is the construction of various enumerative invariants of Calabi-Yau manifolds. They appeared in the counting formula of CDGP. This development has led to a proof of the mirror symmetry conjecture by independent works of Givental $[\mathbf{1 1 0}, \mathbf{2 9}, \mathbf{2 3 2}]$ and of Lian-Liu-Yau [195].

In 1994, Kontsevich [164] expanded and formulated his version of mirror symmetry as an equivalence between complex and symplectic geometry of Calabi-Yau manifolds in all dimension.

The geometric approach to mirror symmetry was finally unveiled by Strominger, Yau and Zaslow [265] in their 1997 paper in which they proposed that mirror symmetry is a geometric version of the Fourier transformation along dual special Lagrangian tori fibrations on mirror Calabi-Yau manifolds. This SYZ proposal has guided many research works.

\section{Invariants of Calabi-Yau manifolds}

5.1. Gromov-Witten invariants. GW invariants are enumerative invariants that play an integral part of the Mirror Symmetry conjecture. The GW invariants were introduced by physicists for counting the holomorphic curves in Calabi-Yau threefolds which are needed to calculate worldsheet instanton corrections to the sigma model partiton function. In their paper, CDGP proposed a formula that counts the number of rational curves of fixed degree on a general quintic Calabi-Yau. (For Calabi-Yau manifolds, Mori theory of rational curves does not apply and it has only be shown by Heath-Brown and Wilson that Calabi-Yau manifolds with Picard number $\rho>13$ must have rational curves. See also $[\mathbf{2 8 5}, \mathbf{2 3 9}$.) Interpreting the CDGP work mathematically, Aspinwall-Morrison [6] realized that the content of the CDGP formula were related to the work of Gromov [119], who first introduced pseudoholomorphic curves to study symplectic geometry, and Witten's work on two-dimensional topological topological field theory $[\mathbf{2 8 7}, \mathbf{2 8 8}]$. Since then, many mathematicians have contributed to the mathematical foundation of this invariants.

There are two mathematical approaches to the problem - one based on symplectic geometry via pseudo-holomorphic maps to symplectic almost complex manifolds and the other based on algebraic geometry and the notion of stable maps. Both are dependent on understanding the gluing formula of WDVV [88], which has been interpreted to be the associative law of quantum cohomology. The rigorous approach to pseudo-holomorphic maps 
which proved Gromov's compactness theorem in full generality is due to Parker-Wolfson [236] and R. Ye [299] based on techniques developed by Sacks-Uhlenbeck [251] and Siu-Yau [260]. Around 1994, Ruan-Tian [250] used pseudo-holomorphic maps to define the symplectic GW invariants for all semipositive manifolds, which include Calabi-Yau manifolds. (The genus zero GW definition was also given by McDuff and Salamon [211].) From the algebro-geometric perspective, Kontsevich-Manin [165] also in 1994 gave an axiomatic treatment of GW classes and their properties for Fano varieties. Kontsevich, then introduced the notion of stable maps in algebraic geometry [163]. Using the moduli of stable maps, Li-Tian [185] and BehrendFantechi $[\mathbf{2 4}, \mathbf{2 3}]$ constructed the virtual cycles of DM-stack with perfect obstruction theories, thus constructing the GW invariants for all smooth projective varieties. The analytical framework for GW invariants was subsequently developed in full generality by Fukaya-Ono, Li-Tian, Ruan, Siebert [103, 186, 249, 255]; some details were clarified later by Zinger [303]. The two approaches give identical invariants, confirmed by Li-Tian and Siebert $[\mathbf{1 8 7}, \mathbf{2 5 6}]$.

5.2. Counting formulas. In 1993-1995, Hosono-Klemm-Theisen-Yau $[\mathbf{1 3 8}, \mathbf{1 3 9}]$ and Hosono-Lian-Yau $[\mathbf{1 4 4}]$ made an interesting observation that was crucial to the understanding of the CDGP counting formula ( $g=0$ mirror symmetry) and its generalizations. They observed that the Picard-Fuchs PDEs that compute the periods of a Calabi-Yau manifold have a "motivic" interpretation. Namely, in order for the classical Frobenius method to yield the periods, the Frobenius parameters must satisfy the cohomological relations on the mirror manifold exactly.

Using a combinatorial recipe of Batyrev and Borisov $[\mathbf{1 3}, \mathbf{1 4}]$ for constructing Calabi-Yau manifolds in toric varieties, Hosono-Klemm-TheisenYau $[\mathbf{1 3 8}, \mathbf{1 3 9}]$ and Hosono-Lian-Yau $[\mathbf{1 4 4}]$ showed that this motivic relation holds true for all such Calabi-Yau manifolds, first for Calabi-Yau complete intersections in weighted projective spaces, then in toric varieties, and finally for noncompact Calabi-Yau manifolds which are sums of line bundles over toric varieties. This motivic relation allowed them to write down the counting formula for a Calabi-Yau manifold easily: it expresses the genus zero GW invariants of a Calabi-Yau manifolds explicitly in terms of the special geometry prepotential of the mirror manifold.

Independently, Candelas-de la Ossa-Font-Katz-Morrison [50, 51] also generalized the CDGP work and gave detail analyses of models of CalabiYau hypersurfaces in weighted projected space with two Kähler parameters.

For positive genus, the Kodaira-Spencer theory of gravity of BershadskyCecotti-Ooguri-Vafa (BCOV) [27] has led to counting formulas for GW invariants of positive genera for many Calabi-Yau manifolds. Hosono-LianYau [144] generalized the BCOV genus-one formula to arbitrary Calabi-Yau complete intersections in toric varieties. Inspired by F-theory and M-theory, 
Klemm-Lian-Roan-Yau [161] have also found a counting formula for GW invariants of Calabi-Yau manifolds of dimension 4 or higher.

More recently, based on the theory of BCOV and the geometry of the moduli of Calabi-Yau threefolds, Yamaguchi and Yau [292] have shown that for the quintic threefold the topological partition functions of all genera can be expressed explicitly as polynomials of five known holomorphic functions. They conjectured that similar polynomials exist for all Calabi-Yau threefolds. As shown by Dijkgraaf [74] in 1995, the BCOV theory applied to an elliptic curve has a close similarity to the theory of quasi-modular forms of Kaneko-Zagier [155]. It has also allowed Huang-Klemm-Quackenbush [146] to calculate the partition function up to genus 51 for the quintic. The discovery by Yamaguchi and Yau has led to renewed interest on quasi-modular forms.

5.3. Proofs of counting formulas for Calabi-Yau threefolds. In 1994, Kontsevich demonstrated [163] that one can approach the GW-invariants of quintics by applying the Atiyah-Bott localization formula to the top Chern classes of vector bundles on the stable map moduli spaces of $\mathbb{C P}^{4}$. Though his method in principle can determine the genus zero GW-invariants of all degrees, more insights are required to settle the mirror conjecture for quintics.

Two independent proofs of the CDGP formula used localization techniques in different ways. One approach based on quantum differential equation in the case of the quintics was due to Givental [110] in 1996, and was later expanded and clarified by others $[\mathbf{2 9}, \mathbf{2 3 2}]$ in 1998. An independent approach based on functorial localization was given by Lian-Liu-Yau [195] in 1997; they later generalized their work to complete intersections in toric varieties in 1999. (See [194] for a comparison of the two approaches.) The theory developed in $[\mathbf{1 9 5}, \mathbf{1 9 6}, \mathbf{1 9 7}]$ - which is called the mirror principle - have been applied to many other generalizations of the CDGP formula. By varying the possible $\mathrm{K}$-classes and evaluating their Chern classes, their approach has also led to a number of new counting formulas for noncompact Calabi-Yau manifolds [195].

To prove the BCOV counting formula for higher genus GW invariants of quintics, a new localization formula for virtual fundamental classes had to be developed. This localization formula was worked out by $\mathrm{Li}$ and Zinger [190] for complete intersection of projective spaces; the genus-one formula of BCOV for quintic Calabi-Yau was subsequently proved by Zinger [304].

5.4. Integrability of mirror map and arithmetic applications. Mirror symmetry has many interesting and often unexpected connections and applications to mathematics. For instance, it is conjectured that near a certain large complex structure limit, the moduli space of a Calabi-Yau manifold admits certain special coordinates. Lian-Yau [198] conjectured that the power series expansion of the mirror map in these coordinates always have 
integer coefficients. These expansions depend on some choices, but the integrality seems to be independent of such choices. This integrality conjecture has been proved for quintics and several other Calabi-Yau threefolds with $h^{1,1}=1$, and a number of isolated examples with $h^{1,1}>1$.

As Lian-Yau [199] showed, mirror maps in some way can be thought of as generalization of modular functions. The precise conditions under which it is is a modular function were determined by Doran in [85]. It is easy to see that the elliptic modular function $j(\tau)$ is nothing but the mirror map for elliptic curves. $j(\tau)$ satisfies a Schwarzian differential equation $\{j(\tau), \tau\}=Q(j)$, where $Q(j)$ is a certain rational function. And in fact, $j$ can be uniquely determined by the differential equation. For certain families of K3 surfaces, Clingher-Doran-Lewis-Whitcher [68] derived the Schwarzian differential equation directly from geometry by studying the Picard-Fuchs equations over modular curves. Indeed, modularity of the mirror map implies integrality, and hence results for families of elliptic curves and K3 surfaces of generic Picard rank 19. However, only a handful of specially constructed families of Calabi-Yau threefolds have classically modular mirror maps.

Klemm-Lian-Roan-Yau [160] have also shown that mirror maps too satisfy similar, but higher order, nonlinear differential equations. These equations can be used to study divisibility property of the instanton numbers of Calabi-Yau threefolds. For example, it was shown that the instanton number $n_{d}$ predicted by the CDGP formula is divisible by 125 (at least for all $d$ coprime to 5 ). If $n_{d}$ correctly counts the number of smooth rational curves in a general quintic, as expected, then the divisibility property of $n_{d}$ above supports a conjecture of Clemens. On another front, the mirror principle, developed by Lian-Liu-Yau $[\mathbf{1 9 5}, \mathbf{1 9 6}, \mathbf{1 9 7}]$ also has important application in birational geometry. For example, Lee-Lin-Wang [177] have used the mirror principle recently to study local models of Calabi-Yau manifolds in their study of analytic continuations of quantum cohomology rings under flops.

Arithmetic properties of algebraic Calabi-Yau manifolds defined over finite fields and their mirrors have been studied. Focusing on the oneparameter $\psi$ family of Fermat quintic threefolds $X_{\psi}$, Candelas, de la Ossa and Rodriguez-Villegas $[\mathbf{5 3}, \mathbf{5 4}]$ showed that the number of $\mathbb{F}_{p}$-rational points can be computed in terms of the periods of the holomorphic threeform. They also found a closed form for the congruence zeta function which counts the number $N_{r}\left(X_{\psi}\right)$ of $\mathbb{F}_{p^{r}}$-rational points. The zeta function is a rational function and the degrees of the numerator and denominator are exchanged between the zeta functions of $X_{\psi}$ and their mirror $Y_{\psi}$. Interestingly, Wan [282] has proved that $N_{r}\left(X_{\psi}\right)=N_{r}\left(Y_{\psi}\right)\left(\bmod p^{r}\right)$ for arbitrary dimension Fermat Calabi-Yau manifolds and has conjectured that such relations should hold for all mirror pair Calabi-Yau manifolds in general.

5.5. Donaldson-Thomas invariants. Another duality on Calabi-Yau threefolds is based on the invariants introduced by Donaldson-Thomas [84]. Paired with the holomorphic three-forms on Calabi-Yau threefolds, 
Donaldson-Thomas introduced and studied the holomorphic Chern-Simons functional on the space of connections on vector bundles over Calabi-Yau threefolds. Their study leads to a collection of new invariants of Calabi-Yau threefolds, modulo some analytical technicality. These technicality can be by-passed in algebraic geometry using the moduli of stable sheaves and their virtual cycles.

A special case is the moduli of rank one stable sheaves. This leads to the virtual counting of ideal sheaves of curves, which are referred to as Donaldson-Thomas invariants. (These invariants based on ideal sheaves of curves can be generalized to all smooth threefolds.) In [215], based on their explicit computation of such invariants for toric threefolds, MaulikNekrasov-Okounkov-Pandharipande (MNOP) conjectured that (the rank one version of) Donaldson-Thomas invariants is, in explicit form, equivalent to the GW invariants of the same varieties. Henceforth, DonaldsonThomas invariants provide integers underpinning for the rational GW invariants.

Recently, Pandharipande and Thomas $[\mathbf{2 3 4}, \mathbf{2 3 5}]$ found a third curvecounting theory involving stable pairs. In order to define how to count these, one must think of curves as defining elements in the derived category of coherent sheaves, where they differ from the ideal sheaves of [215] by a wall crossing in the space of stability conditions [39]. The more transparent geometry has made this curve-counting easier to study, leading to progress [235] on a mathematical definition of the remarkable BPS invariants of Gopakumar-Vafa $[\mathbf{1 1 2}, \mathbf{1 1 3}]$, which give perhaps the best integer description of GW theory for threefolds.

The interaction of the MNOP duality with mirror symmetry is a little mysterious. It relates GW invariants, which belong to the A-model of mirror symmetry, to counting objects of the derived category (which describes the B-model) on the same manifold rather than its mirror. The point is that these latter invariants are independent of complex structures (they are deformation invariant), but depend on the stability conditions, one would hope that such invariants are symplectic invariants in nature, like GW invariants. A purely symplectic construction of the gauge-theoretic invariants of Donaldson-Thomas would be an important advance in our understanding. Mirror symmetry would then relate this derived category picture to the Fukaya category of the mirror. Counting stable sheaves gets replaced by counting special Lagrangians, as proposed by Joyce [151]. His counts are invariant under deformations of symplectic structures, but undergo wall crossings as the complex structure varies.

From physical considerations, Denef and Moore [73] have independently found formulas describing the wall crossing phenomena. They are important for the counting of BPS D-branes bound states in string theory. Specifically, Donaldson-Thomas invariants have been identified with the counting of bound states of a single D6-brane with D2- and D0-branes. Wall crossings are also relevant for making precise the Ooguri-Strominger-Vafa conjecture 
[227] which relates the topological string partition function with BPS Dbranes/black holes degeneracies. At the moment, wall crossing is a subject of much interest in both mathematics and physics, see for example $[\mathbf{1 6 8}, \mathbf{1 0 6}]$.

5.6. Stable bundles and sheaves. Stable holomorphic bundles and sheaves are important geometric objects on Calabi-Yau manifolds and give interesting invariants (e.g. Donaldson-Thomas invariants). Stable principal G-bundles are also necessary data for heterotic strings on Calabi-Yau manifolds and for various duality relations in string theory. The stability condition of Mumford-Takemoto and of Gieseker on sheaves ensures that the moduli space is quasi-projective. By the results of Narasimhan-Seshadri [226] for Riemann surfaces, and Donaldson [79], Uhlenbeck-Yau [277] for higher dimensions, there exist on stable (and poly-stable) bundles connections that solve the Hermitian-Yang-Mills equations. These equations are important for physical applications and requires that the $(2,0)$ and $(0,2)$ part of the curvature two-form vanish and the $(1,1)$ part is traceless.

In dimension one, the classification of vector bundles on an elliptic curve was due to Atiyah [7]. The set of isomorphism classes of indecomposable bundles of a fixed rank and degree is isomorphic to the elliptic curve. For general structure groups, Looijenga [205] and Bernstein-Shvartsman [25] showed that the moduli space of semistable $G$ bundles for any simply-connected group $G$ of rank $r$ is a weight projective space of dimension $r$.

In dimension two, Mukai $[\mathbf{2 2 4}, \mathbf{2 2 5}]$ studied in depth the moduli space $\mathcal{M}^{H}(v)$ of Gieseker-semistable sheaves $F$ on a smooth projective K3 surface $(S, H)$. He showed that in case the moduli space $\mathcal{M}^{H}(v)$ is smooth, it is symplectic. His insight also led to the powerful Fourier-Mukai transformation.

Friedman-Morgan-Witten $[\mathbf{9 5}, \mathbf{9 6}, 97]$ constructed stable principal G-bundles on elliptic Calabi-Yau threefolds (see also Donagi [77] and Bershadsky-Johansson-Pantev-Sadov [28].) The construction is based on spectral covers $[\mathbf{7 8}]$ introduced on curves by Hitchin $[\mathbf{1 3 1}, \mathbf{1 3 2}]$. The spectral data consists of a hypersurface and a line bundle over it. The spectral cover construction can be interpreted in terms of a relative Fourier-Mukai transformation and have been used extensively in string theory (see, for example $[\mathbf{3 1}, \mathbf{3 6}, \mathbf{4}]$ and references therein).

Thomas [267], Andreas, Hernández Ruipérez and Sánchez Gómez [5] have constructed stable bundles on K3 fibration Calabi-Yau threefolds.

5.7. Yau-Zaslow formula for K3 surfaces. In 1996, Yau and Zaslow [298] discovered a formula for the number of rational curves on K3 surfaces in terms of a quasi-modular form. Their method was inspired by string theory considerations.

Let $X$ be a K3 surface. Suppose $C$ is a holomorphic curve in $X$ representing a cohomology class $[C]$. We write its self-intersection number as $[C] \cdot[C]=2 d-2$ and its divisibility, or index, as $r$. If $C$ is a smooth curve, then $d$ is equal to the genus of $C$ and also to the dimension of the linear 
system of $C$. If we denote the number of genus $g$ curves in $X$ representing $[C]$ as $N_{g}(d, r)$. Then the Yau-Zaslow formula says that when $g=0$ they are given by the following formula,

$$
\sum_{d \geq 0} N_{0}(d, r) q^{d}=\prod_{d \geq 1}\left(\frac{1}{1-q^{d}}\right)^{24} .
$$

The Yau-Zaslow formula was generalized by Göttsche [114] to arbitrary projective surface. The universality for having such a formula for all surfaces was analyzed by Liu [200] using Seiberg-Witten theory which is related to the curve counting problem by the work of Taubes on $G W=S W$.

The conjecture originated from a study by Yau and Zaslow on the BPS states in string theory on complex two dimensional Calabi-Yau manifolds, which are K3 surfaces. Shortly after the paper by Yau-Zaslow, Beauville [19], and later Fantechi-Göttsche-van Straten [92], rephrased and clarified the argument of Yau-Zaslow in algebraic geometry for primitive class. Chen [65] in 2002 proved that rational curves of primitive classes in general polarized K3 surfaces are nodal. Combined, these prove the Yau-Zaslow formula for primitive classes.

The Yau-Zaslow formula is for all index $r \geq 1$. Following the original approach of Yau-Zaslow, Li-Wu [188] proved the conjecture for nonprimitive classes of index at most five under the assumption that all rational curves are nodal.

Via a different approach, Bryan and Leung [41] proved the formula for the primitive case by considering elliptic K3 surfaces with section by computing the family GW invariants for the twistor family. These invariants are typically difficult to compute and they used a clever matching method to transport it to an enumerative problem for rational surfaces and then used Cremona transformations to further simplify it. Their method is more powerful than the sheaf-theoretic approach in that it works for any genus as well.

Using a degeneration for the family GW invariants, J.H. Lee-Leung settled the $r=2$ case of the Yau-Zaslow formula [174] and the genus one formula $[\mathbf{1 7 5}]$.

Recently Klemm, Maulik, Pandharipande and Scheidegger [159] proved the Yau-Zaslow formula for any classes by studying a particular Calabi-Yau threefold $M$ with a K3 fibration. The Yau-Zaslow number can be related to the GW invariants on $M$ representing fiber classes. Using localization techniques to compute these threefold invariants they proved the Yau-Zaslow formula.

5.8. Chern-Simons knot invariants, open strings and string dualities. Calabi-Yau geometry is the central object in string duality to unify different types of string theory. Mirror symmetry is just the duality between IIA and IIB string theory as discussed above. Using string duality 
between the large $N$ Chern-Simons theory and the topological string theory of non-compact toric Calabi-Yau manifolds, string theorists have made many striking conjectures about the moduli spaces of Riemann surfaces, ChernSimons knot invariants and GW invariants. Of note are two which have been rigorously proven. First, the Mariño-Vafa conjecture [212] which expresses the generating series of triple Hodge integrals on moduli spaces of Riemann surfaces for all genera and any number of marked points in terms of the Chern-Simons knot invariants was proved by C.-C. Liu-K. Liu-Zhou in $[\mathbf{2 0 1}]$. Second, the Labastilda-Mariño-Ooguri-Vafa conjecture $[\mathbf{2 2 9 ,} \mathbf{1 7 3}$, 172] which predicts integral and algebraic structures of the generating series of the $S U(N)$ Chern-Simons quantum knot invariants was proved by Liu-Peng [203].

GW invariants for all genera and all degrees can be explicitly computed for non-compact toric Calabi-Yau manifolds via the theory of topological vertex. In [2], Aganagic, Klemm, Mariño and Vafa proposed a theory to compute GW invariants in all genera and all degrees of any smooth noncompact toric Calabi-Yau threefold. In that paper, they first postulated the existence of open GW invariants that count holomorphic maps from bordered Riemann surfaces to $\mathbb{C}^{3}$ with boundaries mapped to Lagrangian submanifolds, which they called the topological vertex; they then argued based on a physically derived duality between Chern-Simons theory and GW theory that the topological vertex can be expressed in terms of the explicitly computable Chern-Simons link invariants. Then by a gluing algorithm, they derived an algorithm computing all genera GW invariants of toric CalabiYau threefolds.

In $[\mathbf{1 8 4}]$, J. Li, C.-C. Liu, K. Liu and J. Zhou (LLLZ) developed the mathematical theory of the open GW invariants for toric Calabi-Yau threefold. (In the case compact Calabi-Yau threefolds, open GW invariants have only been defined in the case where the Lagrangian submanifold is the fixed point set of an antiholomorphic involution [259]. See $[\mathbf{2 8 0}, \mathbf{2 3 3}]$ for calculations of open GW invariants on the Calabi-Yau quintic.) The definition of LLLZ relies on applying the relative GW invariants of $\mathrm{J}$. $\mathrm{Li}[\mathbf{1 8 2}, \mathbf{1 8 3}]$ to formal toric Calabi-Yau threefolds. By degenerating a formal toric Calabi-Yau to a union of simple ones, they derived an algorithm that expresses the open GW invariants of any (formal) toric Calabi-Yau in terms of that of the simple one. Their results express the open GW invariants in terms of explicit combinatorial invariants related to the Chern-Simons invariants. In many cases their combinatorial expressions coincide with those of [2], and they conjectured that the two combinatorial expressions should be equal in general. Later, a proof of this conjecture appeared in the work of Maulik-OblomkovOkounkov-Pandharipande [216]. Combined, all genera GW invariant for toric Calabi-Yau threefolds is solved. By using the results of [184], Peng [237] was able to prove the integrality conjecture of Gopakumar-Vafa for all formal toric Calabi-Yau manifolds. 
When applying the mirror principle to certain toric Calabi-Yau manifolds, we get the local mirror formulas of Chiang-Klemm-Yau-Zaslow [66] which are closely related to geometric engineering in string theory [153]. This is an important technique to recover gauge theory such as the SeibergWitten theory at various singularities in the moduli space of string theory [154]. Chiang-Klemm-Yau-Zaslow [66] also studied the asymptotic growth of genus zero Gromov-Witten invariants as the degree runs to infinity. Computational evidences have suggested in many cases a relationship between these growth rates and special values of L-functions. These observations have now been geometrically explained by Doran-Kerr [86], who showed, using higher Abel-Jacobi maps, that they follow from the deep mathematical conjectures of Beilinson-Hodge and Beilinson-Bloch.

\section{Homological mirror symmetry}

The Homological Mirror Symmetry (HMS) conjecture was made in 1994 by Maxim Kontsevich [164]. This was a proposal to give an explanation for the phenomena of mirror symmetry. This conjecture, very roughly, can be explained as follows. Let $\mathrm{X}$ and $\mathrm{Y}$ be a mirror pair of Calabi-Yau manifolds. We view $\mathrm{X}$ as a complex manifold and $\mathrm{Y}$ as a symplectic manifold. The idea is that mirror symmetry provides an isomorphism between certain aspects of complex geometry on $\mathrm{X}$ and certain aspects of symplectic geometry on Y.

More precisely, Kontsevich suggested that the bounded derived category of coherent sheaves on $\mathrm{X}$ is isomorphic to the Fukaya category of $\mathrm{Y}$. The first object has been well-studied, and is known to capture a significant amount of information about the complex geometry on X, while the Fukaya category is a much less familiar object introduced by Fukaya [100] in a 1993 paper. This is not a true category, but something known as an $A_{\infty}$ cateogry: the composition of morphisms is not associative, but only associative up to homotopy. The Fukaya category captures information about the symplectic geometry of $\mathrm{Y}$. Its objects are Lagrangian submanifolds of $\mathrm{Y}$ and morphisms come from intersection points of Lagrangian submanifolds. Compositions involve counting holomorphic disks, and essentially arise from the product in Floer homology.

The homological mirror symmetry conjecture has remained an imposing problem. There have been a number of different threads of work devoted to this. Work of a number of researchers, especially Polishchuk and Zaslow [242] and Fukaya [101], dealt with the simplest cases, namely mirror symmetry for elliptic curves and abelian varieties, respectively. Other work has been devoted to clarifying the conjecture: at first sight, the two categories cannot be isomorphic since the derived category is an actual triangulated category, while the Fukaya category is not an actual category and is not likely to be triangulated. There are various ways around these issues, and there are now precise rigorous statements. Most significantly, the work of Seidel [254] has proved the conjecture for quartic surfaces in projective three-space. 
The HMS conjecture implies that complex manifolds which have equivalent bounded derived categories are mirrored to the same manifold. These manifolds, related by Fourier-Mukai transforms, are called Fourier-Mukai partners. In complex dimension one, Orlov [230] has determined both the group of autoequivalences and the Fourier-Mukai partners of an abelian variety. Interesting results have also known for $K 3$ surfaces. Mukai [224] long ago showed that the Fourier-Mukai partners of a given K3 surface is again a K3. The Fourier-Mukai transform induces a Hodge isometry of the "Mukai lattice" of $K 3$ [231]. Bridgeland and Maciocia [40] have shown that the number of Fourier-Mukai partners of any given K3 is finite. Hosono, Lian, Oguiso, and Yau [142] have recently, given an explicit counting formula for this number. A similar formula was given for abelian surfaces and was used to answer an old question of T. Shioda [140]. They have also given a description for the group of autoequivalences of the bounded derived category of a K3 surface [141]. It turns out that the Fourier-Mukai number formula is closely related to the class numbers of imaginary quadratic fields of prime discriminants [142]. There is also a nice analogue for real quadratic fields. As shown in [143], the real case turns out to be crucial for classifying $c=2$ rational toroidal conformal field theory in physics.

The HMS conjecture for Calabi-Yau manifolds has been generalized to Fano varieties. For toric varieties, the work of Abouzaid [1] established part of the conjecture and was recently settled by Fang-Liu-Treumann-Zaslow [91]. Moreover, for surfaces, Auroux-Katzarkov-Orlov $[\mathbf{9}, \mathbf{1 0}]$ have proved the HMS conjecture for some toric surfaces (i.e. weighted projective planes, Hirzebruch surfaces, and toric blowups of $\mathbb{P}^{2}$ ) and also non-toric del Pezzo surfaces.

Another thread has been addressing the question of how more traditional aspects of mirror symmetry, such as holomorphic curve counting, would follow from homological mirror symmetry.

\section{SYZ geometric interpretation of mirror symmetry}

7.1. Special Lagrangian submanifolds in Calabi-Yau manifolds. By the Wirtinger formula for Kähler manifolds, every complex submanifold in $X$ is absolute volume minimizing. This is a special case of calibration, a notion introduced by Harvey and Lawson [128] in analyzing area-minimizing subvarieties, and later on rediscovered in physics by Becker-BeckerStrominger [21] from supersymmetry considerations. Special Lagrangian submanifolds in Calabi-Yau manifolds form another class of examples of

calibrated submanifolds. A real $n$-dimensional submanifold $L$ in $X$ is called special Lagrangian if the restrictions of both $\omega$ and $\operatorname{Im} \Omega$ to $L$ are zero:

$$
\left.\omega\right|_{L}=\left.\operatorname{Im} \Omega\right|_{L}=0
$$


As calibrated submanifolds, special Lagrangian submanifolds are always absolute volume minimizing.

7.2. The SYZ conjecture - SYZ transformation. In string theory, each Calabi-Yau threefold $X$ determines two twisted theories, one $A$-model and another $B$-model. The mirror symmetry between $X$ and its mirror $Y$ interchanges the two models between them. From the mathematical perspective, A-model is about the symplectic geometry of $X$ and B-model is about the complex geometry of $Y$.

$$
\begin{gathered}
\text { A-model on } X \\
\text { (symplectic geometry) }
\end{gathered} \quad \overleftrightarrow{\text { mirror symmetry }} \begin{gathered}
\text { B-model on } Y \\
\text { (complex geometry) }
\end{gathered}
$$

The search for the underlying geometric root of this symmetry led Strominger, Yau and Zaslow to their conjecture.

In 1996, Strominger, Yau and Zaslow [265] proposed that for a mirror pair $(X, Y)$ that is near a large volume/complex structure limit,

(1) both admit special Lagrangian torus fibrations with sections:

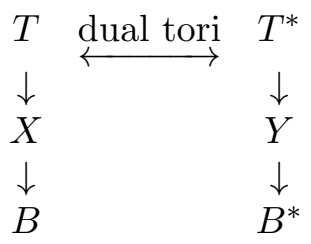

(2) the two torus fibrations are dual to each other;

(3) a fiberwise Fourier-Mukai transformation along fibers interchanges the symplectic (resp. complex) geometry on $X$ with the complex (resp. symplectic) geometry on $Y$.

This is called the $S Y Z$ mirror transformation.

On the nutshell, it says that the mysterious mirror symmetry is simply a Fourier transform. The quantum corrections, for instance the GW invariants, come from the higher Fourier modes. The SYZ conjecture inspired a flourish of work to understand mirror symmetry, which include works of Gross (and with Siebert) $[\mathbf{1 2 2}, \mathbf{1 2 3}, \mathbf{1 2 4}, \mathbf{1 2 5}, \mathbf{1 2 6}]$, Joyce $[\mathbf{1 5 0}, \mathbf{1 5 2}]$, KontsevichSoibelman $[\mathbf{1 6 6}, \mathbf{1 6 7}]$, Vafa [278], Leung-Yau-Zaslow [180] and many others. On the other hand, it has led to new developments of other branches of mathematics, including the calibrated geometry of special Lagrangian submanifolds and the affine geometry with singularities. The work of Auroux has shed some lights on the phenomenon of quantum corrections [8].

7.3. Special Lagrangian geometry. Special Lagrangian submanifolds coupled with unitary flat bundles are branes in A-model in string theory. These geometric objects are crucial to the understanding of the SYZ conjecture. So far, many examples were constructed using cohomogeneity one method by Joyce [150], using singular perturbation method by 
Butscher [44], Lee [176], Haskins-Kapouleas [129] and others. Their deformations are studied by McLean [217]; their moduli spaces by Hitchin [133]; their existence by Schoen-Wolfson [253] using variational approach and by Smoczyk and M.-T. Wang [261] using mean curvature flow. Thomas-Yau [268] formulated a conjecture on the existence and uniqueness of special Lagrangian submanifolds which is the mirror of the theorem of Donaldson, Uhlenbeck and Yau $[\mathbf{7 9}, \mathbf{2 7 7}]$ of the existence of unique Hermitian YangMills connection on any stable holomorphic vector bundle.

7.4. Special Lagrangian fibrations. SYZ conjecture predicts that mirror Calabi-Yau manifolds should admit dual torus fibrations whose fibers are special Lagrangian submanifolds, possibly with singularities.

Lagrangian fibrations is an important notion in symplectic geometry as real polarizations, as well as in dynamical system as completely integrable systems. Their smooth fibers admit canonical integral affine structures and therefore they must be tori in the compact situation. Toric varieties $\mathbb{P}_{\Delta}$, for instance $\mathbb{C} \mathbb{P}^{n+1}$, are examples of Lagrangian fibrations in which the fibers are orbits of an Hamiltonian torus action and the base is a convex polytope $\Delta$.

A complex hypersurface $X=\{f=0\}$ in $\mathbb{C P}^{n+1}$ is a Calabi-Yau manifold if $\operatorname{deg} f=n+2$. The most singular ones is when $X$ is a union of coordinate hyperplanes in $\mathbb{C P}^{n+1}$, which is an example of the large complex structure limit. Such limiting points on the moduli space are important and an explicit construction of them for Calabi-Yau toric hypersurfaces as $T$-fixed points on the moduli space has been given by Hosono-Lian-Yau [145]. A numerical criterion for the large complex structure limit in any one parameter family of Calabi-Yau manifolds has also been given by Lian-Todorov-Yau [193]. At this most singular limit, $X$ inherits a torus fibration from the toric structure on $\mathbb{C P}^{n+1}$. Thus one can try to perturb this to obtain Lagrangian fibration structures on nearby smooth Calabi-Yau manifolds. This approach was carried out by Gross [124], Mikhalkin [219], Ruan [247, 248] and Zharkov [302]. This approach can be generalized to Calabi-Yau hypersurfaces $X$ in any Fano toric variety $\mathbb{P}_{\Delta}$. Furthermore, their mirror manifolds $Y$ are CalabiYau hypersurfaces in another Fano toric variety $\mathbb{P}_{\nabla}$ whose defining polytope is the polar dual to $\Delta$.

The situation is quite different for Calabi-Yau twofolds, namely K3 surfaces, or more generally for hyperkähler manifolds. In this case, the CalabiYau metric on $X$ is Kähler with respect to three complex structures $I, J$ and $K$. When $X$ admits a $J$-holomorphic Lagrangian fibration, then this fibration is a special Lagrangian fibration with respect to the Kähler metric $\omega_{I}$, as well as $\omega_{K}$. Furthermore, SYZ also predicts that mirror symmetry is merely a twistor rotation from $I$ to $K$ in this case. For K3 surfaces, there are plenty of elliptic fibrations and they are automatically complex Lagrangian fibrations because of their low dimension. Furthermore Gross and Wilson [127] described the Calabi-Yau metrics for generic elliptic K3 surfaces by using the singular perturbation method. They used model metrics which 
were constructed by Greene, Shapere, Vafa and Yau [121] away from singular fibers and by Ooguri and Vafa [228] near singular fibers.

7.5. The SYZ transformation. Recall that SYZ conjecture says that mirror symmetry is a Fourier-Mukai transformation along dual special Lagrangian torus fibrations. We also need to include a Legendre transformation on the base affine manifolds. This SYZ transformation was generalized to the mirror symmetry for local Calabi-Yau manifolds by Leung-Vafa [179].

On the mathematical side, Leung-Yau-Zaslow [180] and Leung [178] used the SYZ transformation to verify various correspondences between symplectic geometry and complex geometry between semi-flat Calabi-Yau manifolds when there is no quantum corrections. To include quantum corrections in the SYZ transformation for Calabi-Yau manifolds is a more difficult problem. In the Fano case, there are recent results on applying the SYZ transformation with quantum corrections by Auroux [8], Chan-Leung [63] and Fang $[\mathbf{9 0}]$.

7.6. The SYZ conjecture and tropical geometry. Work of Joyce [152] forced a rethinking of the SYZ conjecture in a limiting setting. The SYZ mirror transformation is now believed to be applicable near the large complex structure limit points. Two groups of researchers, Gross and Wilson [127] on the one hand and Kontsevich and Soibelman [166] on the other, suggested that near a large complex structure limit of n-dimensional Calabi-Yau manifolds, the Ricci-flat metric on the Calabi-Yau manifold converges (in a precise sense known as Gromov-Hausdorff convergence) to an n-dimensional sphere. For example, in the simplest case of an elliptic curve (a real two-dimensional torus), the torus gets thinner as the large complex structure limit is approached, until it converges to a circle. Therefore, the idea is that in the large complex structure limit, the SYZ fibration is expected to be better behaved though the fibers of the SYZ fibration will collapse, with its volume going to zero in the limit.

In any event, once one has this picture of a collapsing fibration, one can ask for a description of the behavior of holomorphic curves in the fibration as the fibres collapse. The expectation is that a holomorphic curve converges to a piecewise linear graph on the limiting sphere. This graph should satisfy certain conditions which turn this graph into what is now known as a "tropical curve." This terminology arises from the "tropical semiring", which is the semiring consisting of real numbers, with addition given by maximum and multiplication given by the usual addition. Tropical varieties are then defined by polynomials over the tropical semiring, and the "zeroes" of a tropical polynomial are in fact points where the piecewise linear function defined by the tropical polynomial is not smooth. This gives rise to piecewise linear varieties, and tropical curves arising as limits of holomorphic curves are examples of such. 
This picture began to emerge in the works of Fukaya [102], Kontsevich and Soibelman [166] around 2000. In particular, Kontsevich's suggestion that one could count holomorphic curves by counting tropical curves was realized in 2003 by Mikhalkin [220], when he showed that curves in toric surfaces could be counted using tropical geometry.

For the purposes of mirror symmetry, it is then important to understand how tropical geometry arises on the mirror side. The initial not so rigorous work of Fukaya in 2000 gave some suggestions as to how this might happen in two dimensions. This was followed by the work of Kontsevich and Soibelman [167] in 2004, again in two dimensions, and the work of Gross and Siebert [126] in 2007 in all dimensions, which demonstrate that the geometry of Calabi-Yau manifolds near large complex structure limits can be described in terms of data of a tropical nature. This provides the clearest link to date between the two sides of mirror symmetry.

\section{Geometries related to Calabi-Yau manifolds}

8.1. Non-Kähler Calabi-Yau manifolds. Given a smooth three dimensional complex manifold $X$ with trivial canonical line bundle, i.e. $K_{X} \cong O_{X}$. When $X$ is Kähler, Yau's theorem [295] provides a unique Ricci-flat Kähler metric in each Kähler class.

A large class of such threefolds which are non-Kähler are obtained by Clemens [67] and Friedman [94] from Calabi-Yau threefolds by an operation called extremal transition or its inverse. An extremal transition is a composition of blowing down rational curves and smoothing the resulting singularity. It has the effect of decreasing the dimension of $H^{2}(X, \mathbb{R})$ and increasing the dimension of $H^{3}(X, \mathbb{R})$ while keeping their sum fixed. For example, the connected sum of $k$ copies of $S^{3} \times S^{3}$ for any $k \geq 2$ can be given a complex structure in this way. Based on this construction, Reid [244] speculated that any two Calabi-Yau threefolds are related by deformations, extremal transitions and their inverses, even though their topologies are different. This speculation demonstrates the potential role of non-Kähler complex manifolds.

It is important to construct canonical metrics on such non-Kähler manifolds which are counterparts of Ricci-flat Kähler metrics on Calabi-Yau manifolds. In 1986, Strominger proposed for supersymmetric compactification in the theory of heterotic string a system of a pair $(\omega, h)$ of a Hermitian metric $\omega$ on a complex three-dimensional manifold $X$ with a non-vanishing holomorphic three form $\Omega$ and a Hermitian metric $h$ on a vector bundle $V$ on $X$. The Strominger system is such a pair satisfying the elliptic system of differential equations,

$$
\begin{gathered}
d\left(\|\Omega\|_{\omega} \omega^{2}\right)=0 \\
F \wedge \omega^{2}=0, \quad F^{2,0}=F^{0,2}=0 \\
4 \sqrt{-1} \partial \bar{\partial} \omega=\alpha^{\prime}\left(\operatorname{Tr}_{T_{X}}\left(R^{2}\right)-\operatorname{Tr}_{E}\left(F^{2}\right)\right),
\end{gathered}
$$


where $R$ (resp. $F$ ) is the curvature of $\omega$ (resp. $h$ ). The first equation is equivalent to the existence of a balanced metric, also the same as the existence of supersymmetry. The system of equations in the second line is the Hermitian-Yang-Mills equations.

When $V$ is the tangent bundle $T_{X}$ and $\omega$ is Kähler, the system is solved by the Calabi-Yau metric. Using perturbation method, J. Li and S.-T. Yau [189] constructed smooth solutions to a class of Kähler Calabi-Yau with irreducible solutions for vector bundles with gauge group $S U(4)$ and $S U$ (5).

The first existence result for solutions of Strominger system for a non-Kähler Calabi-Yau was due to Fu-Yau on a class of torus bundles over K3 surfaces $[\mathbf{9 9}, \mathbf{2 0}]$. (The construction of the complex structure is called the Calabi-Eckmann construction [47] and was carried out by GoldsteinProkushkin [111]. Based on physical arguments of superstring dualities, the existence of such solutions was suggested in $[\mathbf{7 1}, \mathbf{2 2}]$.) Mathematical construction of balanced metrics on manifolds constructed by ClemensFriedman was recently carried out rigorously by Fu-Li-Yau [98].

8.2. Symplectic Calabi-Yau manifolds. Another generalization of Calabi-Yau manifolds are symplectic Calabi-Yau manifolds. Recall a symplectic manifold $(X, \omega)$ is an even dimensional (real) manifold $X$ with $\omega$ a closed, non-degenerate 2 -form on $X$. Examples of symplectic manifolds include Kähler manifolds. Using any compatible almost complex structure on $X$, we can define the first Chern class $c_{1}(X)$ for any symplectic manifold $X$.

Symplectic Calabi-Yau manifolds are symplectic manifolds with $c_{1}(X)=$ 0 . In dimension four, we have the Kodaira-Thurston examples; the homological type of such symplectic manifolds are classified, due to the work of T.-J. $\mathrm{Li}[\mathbf{1 9 2}]$, and to Bauer [17], that their Betti numbers are in the range $b_{1} \leq 4$, $b_{2}^{+} \leq 3$ and $b_{2}^{-} \leq 19$. To their smooth structures, it is conjectured that the diffeomorphism types of such manifolds are either Kähler surfaces with zero Kodaira dimension or oriented torus bundles over torus.

In higher dimensions, Smith-Thomas-Yau [262] has constructed many such examples of symplectic Calabi-Yau manifolds. They contain structures which are mirror to complex non-Kähler Calabi-Yau structures on connected sums of $S^{3} \times S^{3}$. As described in [262], the symplectic mirror of the Clemens-Friedman construction reverses the conifold transition by first collapsing Lagrangian three-spheres and then replacing them by symplectic two-spheres. If one can collapse all three-spheres, then such a process should result in symplectic Calabi-Yau structures on connected sums of $\mathbb{C P}^{3}$.

As the Strominger-Fu-Yau geometry on complex non-Kähler Calabi-Yau manifolds plays an important role in string theory, it is expected to have a dual system on these symplectic Calabi-Yau manifolds which will also play an important role in string theory.

One can also generalize the Ricci-flat condition in dimension four. Donaldson conjectured in [82] that an analogue of the Calabi-Yau theorem should hold on symplectic 4-manifolds. If it is true, there are interesting 
applications to symplectic topology in dimension four. So far relatively little is known about this conjecture, but some progress has been made in [290] and $[\mathbf{2 7 6}]$. There it is shown that the conjecture holds when the manifold is nonnegatively curved, so for example on $\mathbb{C P}^{2}$ with a small perturbation of the standard Kähler structure.

\section{References}

[1] M. Abouzaid, Morse homology, tropical geometry, and homological mirror symmetry for toric varieties. arXiv:math/0610004.

[2] M. Aganagic, A. Klemm, M. Marino and C. Vafa, The topological vertex. Comm. Math. Phys. 254 (2005), no. 2, 425-478.

[3] M. Anderson, P. Kronheimer and C. LeBrun, Complete Ricci-flat Khler manifolds of infinite topological type. Comm. Math. Phys. 125 (1989) 637-642.

[4] B. Andreas and G. Curio, Extension bundles and the standard model. J. High Energy Phys. 0707 (2007), 053.

[5] B. Andreas, D. Hernández Ruipérez, and D. Sánchez Gómez, Stable sheaves over K3 fibrations. arXiv:0802.2903.

[6] P. Aspinwall and D. R. Morrison, Topological field theory and rational curves, Commun. Math. Phys. 15 (1993), 245-262.

[7] M.F. Atiyah, Vector bundles over an elliptic curve. Proc. London Math. Soc. (3) 7 (1957), 414-452.

[8] D. Auroux, Mirror symmetry and T-duality in the complement of an anticanonical divisor. arXiv:0706.3207.

[9] D. Auroux, L. Katzarkov and D. Orlov, Mirror symmetry for del Pezzo surfaces: vanishing cycles and coherent sheaves, Invent. Math. 166 (2006), no. 3, 537-582.

[10] D. Auroux, L. Katzarkov and D. Orlov, Mirror symmetry for weighted projective planes and their noncommutative deformations, Ann. of Math. (2) 167 (2008), no. $3,867-943$.

[11] S. Bando and R. Kobayashi, Ricci-flat Kähler metrics on affine algebraic manifolds. In: Geometry and Analysis on Manifolds (Katata/Kyoto, 1987), Lecture Notes in Math., 1339, Springer, Berlin, 1988, 20-31.

[12] S. Bando and R. Kobayashi, Ricci-flat Kähler metrics on affine algebraic manifolds. II. Math. Ann. 287 (1990), no. 1, 175-180.

[13] V. Batyrev, Dual polyhedra and mirror symmetry for Calabi-Yau hypersurfaces in toric varieties. J. Algebraic Geom. 3 (1994), no. 3, 493-535.

[14] V. Batyrev and L. Borisov, On Calabi-Yau complete intersections in toric varieties. In: Higher-Dimensional Complex Varieties (Trento, 1994), de Gruyter, Berlin, 1996, 39-65.

[15] V. Batyrev, I. Ciocan-Fontanine, B. Kim and D. van Straten, Conifold transitions and mirror symmetry for Calabi-Yau complete intersections in Grassmannians. Nucl. Phys. B 514 (1998), no. 3, 640-666.

[16] V. Batyrev, I. Ciocan-Fontanine, B. Kim and D. van Straten, Mirror symmetry and toric degenerations of partial flag manifolds. Acta Math. 184 (2000), no. 1, 1-39.

[17] S. Bauer, Almost complex 4-manifolds with vanishing first Chern class. J. Differential Geom. 79 (2008), no. 1, 25-32.

[18] A. Beauville, Variétés Kähleriennes dont la première classe de Chern est nulle. J. Differential Geom. 18 (1983), no. 4, 755-782.

[19] A. Beauville, Counting rational curves on K3 surfaces. Duke Math. J. 97 (1999), no. $1,99-108$. 
[20] K. Becker, M. Becker, J.-X. Fu, L.-S. Tseng and S.-T. Yau, Anomaly cancellation and smooth non-Kähler solutions in heterotic string theory. Nucl. Phys. B 751 (2006), $108-128$.

[21] K. Becker, M. Becker and A. Strominger, Five-branes, membranes and nonperturbative string theory. Nucl. Phys. B 456 (1995), 130-152.

[22] K. Becker and K. Dasgupta, Heterotic strings with torsion. J. High Energy Phys. 0211 (2002), 006.

[23] K. Behrend, Gromov-Witten invariants in algebraic geometry. Invent. Math. 127 (1997), no. 3, 601-617.

[24] K. Behrend and B. Fantechi, Intrinsic normal cone. Invent. Math. 128 (1997), no. $1,45-88$.

[25] J.N. Bernstein and O.V. Shvartsman, Chevalleys theorem for complex crystallographic Coxeter groups. Funktsional. Anal. i Prilozhen. 12 (1978), no. 4, 79-80.

[26] M. Bershadsky, S. Cecotti, H. Ooguri and C. Vafa, Holomorphic anomalies in topological field theories. Nuclear Phys. B 405 (1993), no. 2-3, 279-304.

[27] M. Bershadsky, S. Cecotti, H. Ooguri and C. Vafa, Kodaira-Spencer theory of gravity and exact results for quantum string amplitudes. Comm. Math. Phys. 165 (1994), no. 2, 311-427.

[28] M. Bershadsky, A. Johansen, T. Pantev and V. Sadov, On four-dimensional compactifications of F-theory. Nucl. Phys. B 505 (1997), no. 1-2, 165-201.

[29] G. Bini, C. De Concini, M. Polito and C. Procesi, On the work of Givental relative to mirror symmetry. arXiv:math.AG/9805097.

[30] F.A. Bogomolov, Hamiltonian Kähler manifolds. Dolk. Akad. Nauk SSSR 243 (1978), No.5 1101-1104.

[31] V. Bouchard and R. Donagi, An SU(5) heterotic standard model. Phys. Lett. B 633 (2006), no. 6, 783-791.

[32] J-P. Bourguignon, P. Li, Peter and S.-T. Yau, Upper bound for the first eigenvalue of algebraic submanifolds. Comment. Math. Helv. 69 (1994), no. 2, 199-207.

[33] C. P. Boyer and K. Galicki, Sasakian geometry, hypersurface singularities, and Einstein metrics. In: Supplemento ai Rendiconti del Circolo Matematico di Palermo Serie II. Suppl 75 (2005), 57-87.

[34] C. P. Boyer and K. Galicki, Sasakian Geometry, Oxford Mathematical Monographs, Oxford University Press, 2008.

[35] C. P. Boyer, K. Galicki and J. Kollár, Einstein metrics on spheres, Ann. of Math. (2) 162 (2005), 557-580.

[36] V. Braun, Y.-H. He, B.A. Ovrut and T. Pantev, The exact MSSM spectrum from string theory. J. High Energy Phys. 0605 (2006), 043.

[37] V. Braun, T. Brelidze, M.R. Douglas and B.A. Ovrut, Calabi-Yau metrics for quotients complete intersections. J. High Energy Phys. 0805 (2008), 080.

[38] V. Braun, T. Brelidze, M.R. Douglas and B.A. Ovrut, Eigenvalues and eigenfunctions of the scalar Laplace operator on Calabi-Yau manifolds. J. High Energy Phys. 0807 (2008), 120.

[39] T. Bridgeland, Stability conditions on triangulated categories, Ann. of Math. 166 (2007), 317-345.

[40] T. Bridgeland and A. Maciocia, Antony Complex surfaces with equivalent derived categories. Math. Z. 236 (2001), no. 4, 677-697.

[41] J. Bryan and N.C. Leung, The enumerative geometry of K3 surfaces and modular forms. J. Amer. Math. Soc. 13 (2000), no. 2, 371-410.

[42] D. Burns, Y. Hu and T. Luo, HyperKähler manifolds and birational transformations in dimension 4. In: Vector Bundles and Representation Theory (Columbia, MO, 2002), Contemp. Math., 322, Amer. Math. Soc., Providence, RI, 2003, 141-149.

[43] D. Burns and M. Rapoport, On the Torelli problem for Kählerian K3 surfaces. Ann. Sci. École Norm. Sup. (4) 8 (1975), no. 2, 235-273. 
[44] A. Butscher, Regularizing a singular special Lagrangian variety. Comm. Anal. Geom. 12 (2004), no. 4, 733-791.

[45] E. Calabi, On Kähler manifolds with vanishing canonical class. In: Algebraic Geometry and Topology. A Symposium in Honor of S. Lefschetz, Princeton University Press, Princeton, NJ, 1957, 78-89.

[46] E. Calabi, Métriques Kähleriennes et brés holomorphes. Annales Scientiques de 1 École Normale Supérieure, 12 (1979), 268-294.

[47] E. Calabi and B. Eckmann, A class of compact complex manifolds which are not algebraic. Ann. of Math. 58 (1953), 494-500.

[48] P. Candelas, A.M. Dale, C.A. Lutken and R. Schimmrigk, Complete intersection Calabi-Yau manifolds. Nucl. Phys. B 298 (1988), 493-525.

[49] P. Candelas and X. de la Ossa, Comments on conifolds. Nucl. Phys. B 342 (1990), 246-268.

[50] P. Candelas, X. de la Ossa, A. Font, S. Katz and D.R. Morrison, Mirror symmetry for two parameter models - I. Nucl. Phys. B 416 (1994), 481-538.

[51] P. Candelas, X. de la Ossa, A. Font, S. Katz and D.R. Morrison, Mirror symmetry for two parameter models - II. Nucl. Phys. B 429 (1994), 626-674.

[52] P. Candelas, X. de la Ossa, P. Green and L. Parkes, A pair of Calabi-Yau manifolds as an exactly soluble superconformal theory. In: Essays on mirror manifolds, Int. Press, Hong Kong, 1992, 31-95.

[53] P. Candelas, X. de la Ossa and F. Rodriguez-Villegas, Calabi-Yau manifolds over finite fields, I. arXiv:hep-th/0012233.

[54] P. Candelas, X. de la Ossa and F. Rodriguez-Villegas, Calabi-Yau manifolds over finite fields, II. In: Calabi-Yau Varieties and Mirror Symmetry (Toronto, ON, 2001), Fields Inst. Commun., 38, Amer. Math. Soc., Providence, RI, 2003, 121-157.

[55] P. Candelas, P. Green and T. Hübsch, Finite distances between distinct Calabi-Yau manifolds. Phys. Rev. Lett. 62 (1989), 1956-1959.

[56] P. Candelas, P. Green and T. Hübsch, Rolling among Calabi-Yau vacua. Nucl. Phys. B 330 (1990), 49-102.

[57] P. Candelas and A. He: On the number of complete intersection Calabi-Yau Manifolds. Comm. Math. Phys. 135 (1990), 193-200.

[58] P. Candelas, G.T. Horowitz, A. Strominger and E. Witten, Vacuum configurations for superstrings. Nucl. Phys. B 258 (1985), 46-74.

[59] P. Candelas, C.A. Lutken and R. Schimmrigk, Complete intersection Calabi-Yau manifolds. II. Three generation manifolds. Nucl. Phys. B 306 (1988), 113-136.

[60] P. Candelas, M. Lynker and R. Schimmrigk, Calabi-Yau manifolds in weighted $\mathbb{P}^{4}$. Nucl. Phys. B 341 (1990), no. 2, 383-402.

[61] D. Catlin, The Bergman kernel and a theorem of Tian. In: Analysis and Geometry in Several Complex Variables (Katata, 1997), Trends Math., Birkhäuser Boston, Boston, MA, 1999, 1-23.

[62] S. Cecotti and C. Vafa, On classification of $N=2$ supersymmetric theories. Comm. Math. Phys. 158 (1993), no. 3, 569-644.

[63] K.-W. Chan and N.-C. Leung, Mirror symmetry for toric Fano manifolds via SYZ transformations. arXiv:0801.2830.

[64] J. Cheeger and D. Gromoll, The splitting theorem for manifolds of nonnegative Ricci curvature. J. Differential Geometry 6 (1971), 119-128.

[65] X. Chen, A simple proof that rational curves on K3 are nodal. Math. Ann. 324 (2002), no. 1, 71-104.

[66] T.-M. Chiang, A. Klemm, S.-T. Yau and E. Zaslow, Local Mirror Symmetry: Calculations and Interpretations. arXiv:hep-th/9903053.

[67] C.H. Clemens, Double solids. Adv. in Math. 47 (1983), no. 2, 107-230.

[68] A. Clingher, C.F. Doran, J. Lewis and U. Whitcher, Normal forms, K3 surface moduli, and modular parametrizations. arXiv:0712.1880. 
[69] D. Cox and S. Katz, Mirror Symmetry and Algebraic Geometry. Mathematical Surveys and Monographs, 68. American Mathematical Society, Providence, RI, 1999.

[70] X. Dai, K. Liu and X. Ma, On the asymptotic expansion of Bergman kernel. J. Differential Geom. 72 (2006), no. 1, 1-41.

[71] K. Dasgupta, G. Rajesh and S. Sethi, M theory, orientifolds and G-flux. J. High Energy Phys. 9908 (1999), 023.

[72] J.-P. Demailly and J. Kollár, Semi-continuity of complex singularity exponents and Kähler-Einstein metrics on Fano orbifolds. Ann. Sci. Ecole Norm. Sup. (4) 34 (2001), no. $4,525-556$.

[73] F. Denef and G. Moore, Split states, entropy enigmas, holes and halos. arXiv:hep-th/ 0702146.

[74] R. Dijkgraaf, Mirror symmetry and elliptic curves. In: The Moduli Space of Curves (Texel Island, 1994) Progr. Math., 129, Birkhauser Boston, Boston, MA, 1995, 149-163.

[75] R. Dijkgraaf, E. Verlinde and H. Verlinde, Topological strings in $d<1$. Nucl. Phys. B 352 (1991), no. 1, 59-86.

[76] L. Dixon, Some world-sheet properties of superstring compactifications, or orbifolds and otherwise. In: Superstrings, Unified Theories and Cosmology 1987 (Trieste, 1987), ICTP Ser. Theoret. Phys. vol. 4, World Sci., Teaneck, NJ, 1988, 67-126.

[77] R. Donagi, Principal bundles on elliptic fibrations. Asian J. Math. 1 (1997), no. 2, $214-223$.

[78] R. Donagi and E. Markman, Spectral covers, algebraically completely integrable, Hamiltonian systems, and moduli of bundles. In: Integrable systems and quantum groups (Montecatini Terme, 1993), Lecture Notes in Math., 1620, Springer, Berlin, 1996, 1-119.

[79] S. Donaldson, Anti self-dual Yang-Mills connections over complex algebraic surfaces and stable vector bundles. Proc. London Math. Soc. (3) 50 (1985), no. 1, 1-26.

[80] S. Donaldson, Scalar curvature and projective embeddings. I. J. Differential Geom. 59 (2001), no. 3, 479-522.

[81] S. Donaldson, Some numerical results in complex differential geometry, arXiv:math/ 0512625 .

[82] S. Donaldson, Two-forms on four-manifolds and elliptic equations, In: Inspired by S.S. Chern, Nankai Tracts Math., 11, World Sci. Publ., Hackensack, NJ, 2006, 153-172.

[83] S. Donaldson, Extremal metrics on toric surfaces: a continuity method. J. Differential Geom. 79 (2008), no. 3, 389-432.

[84] S. Donaldson and R. Thomas. Gauge theory in higher dimensions. In: The Geometric Universe (Oxford, 1996), Oxford Univ. Press, Oxford, 1998, 31-47.

[85] C.F. Doran, Picard-Fuchs Uniformization and Modularity of the Mirror Map. Comm. Math. Phys. 212 (2000), 625-647.

[86] C.F. Doran and M. Kerr, Algebraic K-theory of toric hypersurfaces. arXiv:0809.4669.

[87] M. R. Douglas, R. L. Karp, S. Lukic and R. Reinbacher, Numerical Calabi-Yau metrics. J. Math. Phys. 49 (2008), 032302.

[88] B. Dubrovin, Integrable systems in topological field theory. Nucl. Phys. B 379 (1992), no. 3, 627-689.

[89] T. Eguchi and A. J. Hanson, Asymptotically at selfdual solutions to Euclidean gravity. Phys. Lett. B 74 (1978), 249-251.

[90] B. Fang, Homological mirror symmetry is T-duality for $\mathbb{P}^{n}$. arXiv:0804.0646.

[91] B. Fang, C.-C. Liu, D. Treumann and E. Zaslow, T-Duality and equivariant homological mirror symmetry for toric varieties. arXiv:0811.1228.

[92] B. Fantechi, L. Göttsche and D. van Straten, Euler number of the compactified Jacobian and multiplicity of rational curves. J. Algebraic Geom. 8 (1999), no. 1, 115-133. 
[93] S. Ferrara, R. Kallosh and A. Strominger, $N=2$ extremal black holes. Phys. Rev. D 52 (1995), 5412-5416.

[94] R. Friedman, Simultaneous resolution of threefold double points. Math. Ann. (1986), no. 4, 671-689.

[95] R. Friedman, J.W. Morgan and E. Witten, Vector bundles and F theory. Comm. Math. Phys. 187 (1997), no. 3, 679-743.

[96] R. Friedman, J.W. Morgan and E. Witten, Principal G-bundles over elliptic curves. Math. Res. Lett. 5 (1998), no. 1-2, 97-118.

[97] R. Friedman, J.W. Morgan and E. Witten, Vector bundles over elliptic fibrations. J. Algebraic Geom. 8 (1999), no. 2, 279-401.

[98] J.-X. Fu, J. Li and S.-T. Yau, Constructing balanced metrics on some families of non-Kähler Calabi-Yau threefolds. arXiv:0809.4748.

[99] J.-X. Fu and S.-T. Yau, The theory of superstring with flux on non-Kähler manifolds and the complex Monge-Ampère equation. J. Differential Geom. 78 (2008), 369-428.

[100] K. Fukaya, Morse homotopy, $A^{\infty}$-category, and Floer homologies. In: Proceedings of GARC Workshop on Geometry and Topology '93 (Seoul, 1993), Lecture Notes Ser., 18, Seoul Nat. Univ., Seoul, 1993, 1-102.

[101] K. Fukaya, Mirror symmetry of abelian varieties and multi-theta functions. J. Algebraic Geom. 11 (2002), no. 3, 393-512.

[102] K. Fukaya, Multivalued Morse theory, asymptotic analysis and mirror symmetry. In: Graphs and patterns in mathematics and theoretical physics, Proc. Sympos. Pure Math., 73, Amer. Math. Soc., Providence, RI, 2005, 205-278.

[103] K. Fukaya and K. Ono, Arnold conjecture and Gromov-Witten invariant. Topology 38 (1999), no. 5, 933-1048.

[104] A. Futaki, Complete Ricci-flat Kähler metrics on the canonical bundles of toric Fano manifolds. arXiv:math/0703138.

[105] A. Futaki, H. Ono and G. Wang, Transverse Kähler geometry of Sasaki manifolds and toric Sasaki-Einstein manifolds. arXiv:math.DG/0607586.

[106] D. Gaiotto, G.W. Moore and A. Neitzke, Four-dimensional wall-crossing via three-dimensional field theory . arXiv:0807.4723.

[107] A. Gathmann, The number of plane conics 5-fold tangent to a given curve. Compos. Math. 141 (2005), no. 2, 487-501.

[108] J. P. Gauntlett, D. Martelli, J. Sparks and D. Waldram, Sasaki-Einstein metrics on $S^{2} \times S^{3}$. Adv. Theor. Math. Phys. 8 (2004), 711-734.

[109] J. P. Gauntlett, D. Martelli, J. Sparks and S.-T. Yau, Obstructions to the existence of Sasaki-Einstein metrics. Commun. Math. Phys. 273 (2007), 803-827.

[110] A. Givental, Equivariant Gromov-Witten invariants. Internat. Math. Res. Notices (1996), no. 13, 613-663.

[111] E. Goldstein and S. Prokushkin, Geometric model for complex non-Kähler manifolds with SU(3) structure. Comm. Math. Phys. 251 (2004), no. 1, 65-78.

[112] R. Gopakumar and C. Vafa, M-theory and topological strings-I. arXiv:hep-th/ 9809187.

[113] R. Gopakumar and C. Vafa, M-theory and topological strings-II. arXiv:hep-th/ 9812127.

[114] L. Göttsche, A conjectural generating function for numbers of curves on surfaces. Comm. Math. Phys. 196 (1998), no. 3, 523-533.

[115] B. Greene and K. Kirklin, On the equivalence of the two most favoured Calabi-Yau compactifications. Comm. Math. Phys. 113 (1987), no. 1, 105-114.

[116] B. Greene and M. Plesser, Duality in Calabi-Yau moduli space. Nucl. Phys. B 338 (1990), no. 1, 15-37.

[117] P. Green and T. Hübsch, Calabi-Yau manifolds as complete intersections in products of projective spaces. Comm. Math. Phys. 109 (1987), 99-108. 
[118] P. Green and T. Hübsch, Connecting moduli spaces of Calabi-Yau threefolds. Comm. Math. Phys. 119 (1988) 431-441.

[119] M. Gromov, Pseudo-holomorphic curves on almost complex manifolds. Invent. Math. 82 (1985) 307-347.

[120] P. Green, T. Hübsch and C. A. Lütken, All the Hodge numbers for all Calabi-Yau complete intersections. Class. Q. Grav. 6 (1989) 105-124.

[121] B. Greene, A. Shapere, C. Vafa and S.-T. Yau, Stringy cosmic strings and noncompact Calabi-Yau manifolds. Nucl. Phys. B 337 (1990), no. 1, 1-36.

[122] M. Gross, Special Lagrangian fibrations. I. Topology. In: Winter School on Mirror Symmetry, Vector Bundles and Lagrangian Submanifolds (Cambridge, MA, 1999), AMS/IP Stud. Adv. Math., 23, Amer. Math. Soc., Providence, RI, 2001, 65-93.

[123] M. Gross, Special Lagrangian fibrations. II. Geometry. A survey of techniques in the study of special Lagrangian fibrations. In: Winter School on Mirror Symmetry, Vector Bundles and Lagrangian Submanifolds (Cambridge, MA, 1999), AMS/IP Stud. Adv. Math., 23, Amer. Math. Soc., Providence, RI, 2001, 95-150.

[124] M. Gross, Topological mirror symmetry. Invent. Math. 144 (2001), no. 1, 75-137.

[125] M. Gross and B. Siebert, Affine manifolds, log structures, and mirror symmetry. Turkish J. Math. 27 (2003), no. 1, 33-60.

[126] M. Gross and B. Siebert, From real affine geometry to complex geometry. arXiv:math/0703822.

[127] M. Gross and P.M.H. Wilson, Large complex structure limits of K3 surfaces, J. Differential Geom. 55 (2000), no. 3, 475-546.

[128] F.R. Harvey and H.B. Lawson, Calibrated geometries. Acta Math. 148, (1982) 47-157.

[129] M. Haskins and N. Kapouleas, Special Lagrangian cones with higher genus links. Invent. Math. 167 (2007), no. 2, 223-294.

[130] D.R. Heath-Brown and P.M.H. Wilson, Calabi-Yau threefolds with $\rho>13$. Math. Ann. 294 (1992), no. 1, 49-57.

[131] N. Hitchin, The self-duality equations on a Riemann surface. Proc. London Math. Soc. (3) 55 (1987), no. 1, 59-126.

[132] N. Hitchin, Stable bundles and integrable systems. Duke Math. J. 54 (1987), no. 1, 91-114.

[133] N. Hitchin, The moduli space of special Lagrangian submanifolds. Ann. Scuola Norm. Sup. Pisa Cl. Sci. (4) 25 (1997), no. 3-4, 503-515.

[134] N. Hitchin, The geometry of three-forms in six dimensions. J. Differential Geom. 55 (2000), no. 3, 547-576.

[135] N. Hitchin, Stable forms and special metrics. In: Global differential geometry: the mathematical legacy of Alfred Gray (Bilbao, 2000), Contemp. Math., 288, Amer. Math. Soc., Providence, RI, 2001, 70-89.

[136] K. Hori, S. Katz, A. Klemm, R. Pandharipande, R. Thomas, C. Vafa, R. Vakil and E. Zaslow, Mirror Symmetry. Clay Mathematics Monographs, 1. American Mathematical Society, Providence, RI; Clay Mathematics Institute, Cambridge, MA, 2003.

[137] K. Hori and C. Vafa, Mirror symmetry. arXiv:hep-th/0002222.

[138] S. Hosono, A. Klemm, S. Theisen and S.-T. Yau, Mirror symmetry, mirror map and applications to Calabi-Yau hypersurfaces. Commun. Math. Phys. 167, (1995), 301-350.

[139] S. Hosono, A. Klemm, S. Theisen and S.-T. Yau, Mirror symmetry, mirror map and applications to complete intersection Calabi-Yau spaces. Nucl. Phys. B 433, (1995), 501-554.

[140] S. Hosono, B. Lian, K. Oguiso and S.-T. Yau, Kummer structures on K3 surface: an old question of T. Shioda. Duke Math. J. 120 (2003), no. 3, 635-647. 
[141] S. Hosono, B. Lian, K. Oguiso and S.-T. Yau, Autoequivalences of derived category of a K3 surface and monodromy transformations. J. Algebraic Geom. 13 (2004), no. $3,513-545$.

[142] S. Hosono, B. Lian, K. Oguiso and S.-T. Yau, Fourier-Mukai number of a K3 surface. Algebraic structures and moduli spaces, 177-192, CRM Proc. Lecture Notes, 38, Amer. Math. Soc., Providence, RI, 2004.

[143] S. Hosono, B. Lian, K. Oguiso and S.-T. Yau, $c=2$ rational conformal field theories via the Gauss product. Comm. Math. Phys. 241 (2003), 245-286.

[144] S. Hosono, B. Lian and S.-T. Yau, GKZ-generalized hypergeometric systems in mirror symmetry of Calabi-Yau hypersurfaces. Comm. Math. Phys. 182 (1996), no. 3, 535-577.

[145] S. Hosono, B. Lian and S.-T. Yau,. Maximal degeneracy points of GKZ systems. J. Amer. Math. Soc. 10 (1997), no. 2, 427-443.

[146] M.X. Huang, A. Klemm and S. Quackenbush, Topological string theory on compact Calabi-Yau: modularity and boundary conditions. arXiv:hep-th/0612125.

[147] T. Hübsch, Calabi-Yau manifolds - motivations and constructions. Commun. Math. Phys. 108 (1987), 291-318.

[148] T. Hübsch, Calabi-Yau Manifolds: A Bestiary for Physicists. World Scientific Publishing Co., Inc., River Edge, NJ, 1992.

[149] D. Huybrechts, Compact hyperkhler manifolds: basic results. Invent. Math. 135 (1999), 63-113.

[150] D. Joyce, Constructing special Lagrangian $m$-folds in $\mathbb{C}^{m}$ by evolving quadrics. Math. Ann. 320 (2001), no. 4, 757-797.

[151] D. Joyce, On counting special Lagrangian homology 3-spheres, Contemp. Math. 314 (2002), 125-151.

[152] D. Joyce, Singularities of special Lagrangian fibrations and the $S Y Z$ conjecture. Comm. Anal. Geom. 11 (2003), 859-907.

[153] S. Katz, A. Klemm and C. Vafa, Geometric engineering of quantum field theories. Nucl. Phys. B 497 (1997), 173-195.

[154] S. Katz, P. Mayr and C. Vafa, Mirror symmetry and exact solution of $4 D N=2$ gauge theories. I. Adv. Theor. Math. Phys. 1 (1997), no. 1, 53-114.

[155] M. Kaneko and D. Zagier, A generalized Jacobi theta function and quasimodular forms. In: The Moduli Space of Curves (Texel Island, 1994), Progr. Math., 129, Birkhuser Boston, Boston, MA, 1995, 165-172.

[156] Y. Kawamata, Unobstructed deformations. A remark on a paper of $Z$. Ran: "Deformations of manifolds with torsion or negative canonical bundle." J. Algebraic Geom. 1 (1992), no. 2, 183-190.

[157] Y. Kawamata, Unobstructed deformations. II. J. Algebraic Geom. 4 (1995), no. 2, $277-279$.

[158] Y. Kawamata, Flops connect minimal model. arXiv:0704.1013.

[159] A. Klemm, D. Maulik, R. Pandharipande and E. Scheidegger, Noether-Lefschetz theory and the Yau-Zaslow conjecture, preprint 2008.

[160] A. Klemm, B. Lian, S.-S. Roan and S.-T. Yau, A note on ODEs from mirror symmetry. In: Functional analysis on the eve of the 21st century, Vol. II (New Brunswick, NJ, 1993), Progr. Math., 132, Birkhuser Boston, Boston, MA, 1996, 301-323.

[161] A. Klemm, B. Lian, S.-S. Roan and S.-T. Yau, Calabi-Yau four-folds for M- and F-theory compactifications. Nucl. Phys. B 518 (1998), no. 3, 515-574.

[162] J. Kollár, Flops. Nagoya Math. J. 113 (1989), 15-36.

[163] M. Kontsevich, Enumeration of rational curves via torus actions. In: The Moduli Space of Curves, Progress in Math. vol. 129, Birkhäuser, 1995, 335-368.

[164] M. Kontsevich, Homological algebra of mirror symmetry. Proceedings of the International Congress of Mathematicians, Vol. 1, 2 (Zurich, 1994), 120-139, Birkhauser, Basel, 1995. 
[165] M. Kontsevich and Yu.I. Manin, Gromov-Witten classes, quantum cohomology, and enumerative geometry. Comm. Math. Phys. 164 (1994), 525-562.

[166] M. Kontsevich and Y. Soibelman, Homological mirror symmetry and torus fibrations. In: Symplectic Geometry and Mirror Symmetry (Seoul, 2000), World Sci. Publ., River Edge, NJ, 2001, 203-263.

[167] M. Kontsevich and Y. Soibelman, Affine structures and non-Archimedean analytic spaces. In: The Unity of Mathematics, Progr. Math., 244, Birkhauser Boston, Boston, MA, 2006, 321-385.

[168] M. Kontsevich and Y. Soibelman, Stability structures, motivic Donaldson-Thomas invariants and cluster transformations. arXiv:0811.2435.

[169] M. Kreuzer and H. Skarke, Complete classification of reflexive polyhedra in four dimensions. Adv. Theor. Math. Phys. 4 (2002), 1209-1230.

[170] M. Kreuzer and H. Skarke, Reflexive polyhedra, weights and toric Calabi-Yau fibrations, Rev. Math. Phys. 14 (2002), 343-374.

[171] V.S. Kulikov, Degenerations of K3 surfaces and Enriques surfaces. Izv. Akad. Nauk SSSR Ser. Mat. 41 (1977), no. 5, 1008-1042.

[172] J.M.F. Labastida and M. Marino, A new point of view in the theory of knot and link invariants. J. Knot Theory Ramifications 11 (2002), 173-197.

[173] J.M.F. Labastida, M. Marino and C. Vafa, Knots, links and branes at large N. J. High Energy Phys. (2000), no. 11, 007.

[174] J. Lee and N.C. Leung, Yau-Zaslow formula on K3 surfaces for non-primitive classes. Geom. Topol. 9 (2005), 1977-2012

[175] J. Lee and N.C. Leung, Counting elliptic curves in K3 surfaces. J. Algebraic Geom. 15 (2006), 591-601.

[176] Y.-I. Lee, Embedded special Lagrangian submanifolds in Calabi-Yau manifolds. Comm. Anal. Geom. 11 (2003), no. 3, 391-423.

[177] Y.-P. Lee, H.-W. Lin and C.-L. Wang, Flops, motives and invariance of quantum rings. arXiv:math.AG/0608370

[178] N.-C. Leung, mirror symmetry without corrections. Comm. Anal. Geom. 13 (2005), no. 2, 287-331.

[179] N.C. Leung, C. Vafa, Branes and toric geometry. Adv. Theor. Math. Phys. 2 (1998), no. $1,91-118$.

[180] N.-C. Leung, S.-T. Yau and E. Zaslow, From special Lagrangian to HermitianYang-Mills via Fourier-Mukai transform. Adv. Theor. Math. Phys. 4 (2000), no. 6, 1319-1341.

[181] W. Lerche, C. Vafa and N.P. Warner, Chiral rings in $N=2$ superconformal theories. Nucl. Phys. B 324 (1989), no. 2, 427-474.

[182] J. Li, Stable Morphisms to singular schemes and relative stable morphisms. J. Differential Geom. 57 (2001), 509-578.

[183] J. Li, A degeneration formula of Gromov-Witten invariants. J. Differential Geom. 60 (2002), 199-293.

[184] J. Li, C.-C. Liu, K. Liu and J. Zhou, A mathematical theory of the topological vertex. arXiv:math/0408426.

[185] J. Li and G. Tian, Virtual moduli cycles and Gromov-Witten invariants of algebraic varieties. J. Amer. Math. Soc. 11 (1998), no. 1, 119-174.

[186] J. Li and G. Tian, Virtual moduli cycles and Gromov-Witten invariants of general symplectic manifolds. In: Topics in Symplectic 4-Manifolds (Irvine, CA, 1996), First Int. Press Lect. Ser., I, Int. Press, Cambridge, MA, 1998, 47-83.

[187] J. Li and G. Tian, Comparison of algebraic and symplectic Gromov-Witten invariants. Asian J. Math. 3 (1999), no. 3, 689-728.

[188] J. Li and B. Wu, Note on a conjecture of Gopakumar-Vafa. Chinese Ann. Math. Ser. B 27 (2006), no. 2, 219-242. 
[189] J. Li and S.-T. Yau, The existence of supersymmetric string theory with torsion. J. Differential Geom. 70 (2005), 143-181.

[190] J. Li and A. Zinger, On genus-one Gromov-Witten invariants of complete intersections. arXiv:math/0507104.

[191] P. Li and S.-T. Yau, A new conformal invariant and its applications to the Willmore conjecture and the first eigenvalue of compact surfaces. Invent. Math. 69 (1982), no. 2, 269-291.

[192] T.-J. Li, Quaternionic bundles and Betti numbers of symplectic 4-manifolds with Kodaira dimension zero. Int. Math. Res. Not. 2006, Art. ID 37385, 28 pp.

[193] B. Lian, A. Todorov and S-T. Yau, Maximal unipotent monodromy for complete intersection CY manifolds. Amer. J. Math. 127 (2005), no. 1, 1-50.

[194] B. Lian and K. Liu, On the mirror conjecture. Posted on http://www.doctoryau.com.

[195] B. Lian, K. Liu and S.-T. Yau, Mirror principle I. Asian J. Math. 1 (1997), no. 4, 729-763.

[196] B. Lian, K. Liu and S.-T. Yau, Mirror principle II. Asian J. Math. 3 (1999), no. 1, 109-146.

[197] B. Lian, K. Liu and S.-T. Yau, Mirror principle III. Asian J. Math. 3 (2000), no. 4, 771-800.

[198] B. Lian and S.-T. Yau, Mirror maps, modular relations and hypergeometric series I. arXiv:hep-th/9507151.

[199] B. Lian and S.-T. Yau, Mirror maps, modular relations and hypergeometric series II. Nucl. Phys. Proc. Suppl. 46 (1996), 248-262.

[200] A.K. Liu, Family blowup formula, admissible graphs and the enumeration of singular curves. I. J. Differential Geom. 56 (2000), no. 3, 381-579.

[201] C.-C. Liu, K. Liu and J. Zhou, A proof of a conjecture of Marino-Vafa on Hodge integrals. J. Differential Geom. 65 (2004), 289-340.

[202] K. Liu and X. Ma, A remark on 'Some numerical results in complex differential geometry'. Math. Res. Lett. 14 (2007), no. 2, 165-171.

[203] K. Liu and P. Peng, Proof of the Labastida-Marino-Ooguri-Vafa Conjecture. arXiv:0704.1526.

[204] R. Livné, Motivic orthogonal two-dimensional representations of $\operatorname{Gal}(\bar{Q} / \mathbf{Q})$. Israel J. Math. 92 (1995), no. 1-3, 149-156.

[205] E. Looijenga, Root systems and elliptic curves. Invent. Math. 38 (1976/77), no. 1, $17-32$.

[206] E. Looijenga, A Torelli theorems for Kähler K3 surfaces. In: Geometry Symposium (Utrecht, 1980), Lecture Notes in Math., 894, 1981, 107-112.

[207] E. Looijenga and C Peters, Torelli theorems for Kähler K3 surfaces. Compositio Math. 42 (1980/81), no. 2, 145-186.

[208] Z. Lu, On the lower order terms of the asymptotic expansion of Tian-Yau-Zelditch, Amer. J. Math. 122 (2000), 235273.

[209] Z. Lu and X. Sun, Weil-Petersson geometry on moduli space of polarized Calabi-Yau manifolds. J. Inst. Math. Jussieu 3 (2004), no. 2, 185-229.

[210] H. Luo, Geometric criterion for Gieseker-Mumford stability of polarized manifolds. J. Differential Geom. 49 (1998), no. 3, 577-599.

[211] D. McDuff and D. Salamon, J-Holomorphic Curves and Quantum Cohomology. American Mathematical Society, Providence, RI, 1994.

[212] M. Mariño and C. Vafa, Framed knots at large N, Orbifolds in Mathematics and Physics (Madison, WI, 2001), Contemp. Math., 310, Amer. Math. Soc., Providence, RI, 2002, 185-204.

[213] D. Martelli, J. Sparks and S.-T. Yau, The geometric dual of a-maximisation for toric Sasaki-Einstein manifolds. Commun. Math. Phys. 268 (2006), 39-65.

[214] D. Martelli, J. Sparks and S.-T. Yau, Sasaki-Einstein Manifolds and Volume Minimisation. Commun. Math. Phys. 280 (2008), 611-673. 
[215] D. Maulik, N. Nekrasov, A. Okounkov and R. Pandharipande, Gromov-Witten theory and Donaldson-Thomas theory. I. Compos. Math. 142 (2006), 1263-1285.

[216] D. Maulik, A. Oblomkov, A. Okounkov and R. Pandharipande, Gromov-Witten/ Donaldson-Thomas correspondence for toric 3-folds. arXiv:0809.3976.

[217] R.C. McLean, Deformations of calibrated submanifolds, Comm. Anal. Geom. 6 (1998), no. 4, 705-747.

[218] M. L. Michelsohn, Kähler manifolds with vanishing first Chern class. In: Seminar on Differential Geometry, Ann. of Math. Stud., 102, Princeton Univ. Press, Princeton, N.J., 1982, 359-361.

[219] G. Mikhalkin, Decomposition into pairs-of-pants for complex algebraic hypersurfaces. Topology 43 (2004), no. 5, 1035-1065.

[220] G. Mikhalkin, Enumerative tropical algebraic geometry in $\mathbb{R}^{2}$. J. Amer. Math. Soc. 18 (2005), no. 2, 313-377

[221] D. R. Morrison, Mirror symmetry and rational curves on quintic threefolds: a guide for mathematicians. J. Amer. Math. Soc. 6 (1993), no. 1, 223-247.

[222] D. Morrison and R. Plesser, Towards mirror symmetry as duality for twodimensional abelian gauge theories. Nucl. Phys. B Proc. Suppl. 46 (1996), 177-186.

[223] G. Moore, Arithmetic and attractors. arXiv:hep-th/9807087.

[224] S. Mukai, Symplectic structure of the moduli space of sheaves on an abelian K3 surface. Invent. Math. 77 (1984), 101-116.

[225] S. Mukai, On the moduli space of bundles on K3 surfaces. I. Vector bundles on algebraic varieties (Bombay, 1984), 341-413, Tata Inst. Fund. Res. Stud. Math., 11, Tata Inst. Fund. Res., Bombay, 1987.

[226] M.S. Narasimhan and C.S. Seshadri, Stable and unitary vector bundles on a compact Riemann surface. Ann. of Math. (2) 82 (1965), 540-567.

[227] H. Ooguri, A. Strominger and C. Vafa, Black Hole Attractors and the Topological String. Phys. Rev. D70 (2004), 106007.

[228] H. Ooguri and C. Vafa, Summing up Dirichlet instantons. Phys. Rev. Lett. 77 (1996), no. 16, 3296-3298.

[229] H. Ooguri and C. Vafa, Knot invariants and topological strings, Nucl. Phys. B 577 (2000), 419-438.

[230] D. Orlov, Equivalences of derived categories and K3 surfaces. Algebraic geometry, 7. J. Math. Sci. (New York) 84, no. 5 (1997), 13611381.

[231] D. Orlov, On equivalences of derived categories of coherent sheaves on abelian varieties. arXiv:math.AG/9712017.

[232] R. Pandharipande, Rational curves on hypersurfaces (after A. Givental). In: Séminaire Bourbaki. Vol. 1997/98. Astrisque No. 252 (1998), Exp. No. 848, 5, 307-340.

[233] R. Pandharipande, J. Solomon and J. Walcher, Disk enumeration on the quintic 3-fold, J. Amer. Math. Soc. 21 (2008), no. 4, 1169-1209.

[234] R. Pandharipande and R. Thomas, Curve counting via stable pairs in the derived category. arXiv:0707.2348.

[235] R. Pandharipande and R. Thomas, Stable pairs and BPS invariants. arXiv: 0711.3899.

[236] T. Parker and J.G. Wolfson, Pseudo-holomorphic maps and bubble trees. J. Geom. Anal. 3 (1993), no. 1, 63-98.

[237] P. Peng, A simple proof of Gopakumar-Vafa conjecture for local toric Calabi-Yau manifolds. Comm. Math. Phys. 276 (2007), no. 2, 551-569.

[238] U. Persson and H. Pinkham, Degeneration of surfaces with trivial canonical bundle. Ann. of Math. (2) 113 (1981), no. 1, 4566.

[239] T. Peternell, Calabi-Yau manifolds and a conjecture of Kobayashi. Math. Z. 207 (1991), no. 2, 305-318. 
[240] S. Piunikhin, Quantum and Floer Cohomology have the same Ring Structure. arXiv:hep-th/9401130.

[241] I. Pjateckii-Šapiro and I. Šafarevič, A Torelli theorem for algebraic surfaces of type K3, Math. USSR Izv. 5 (1971), 547-588.

[242] A. Polishchuk and E. Zaslow, Categorical mirror symmetry: the elliptic curve. Adv. Theor. Math. Phys. 2 (1998), no. 2, 443-470.

[243] Z. Ran, Deformations of manifolds with torsion or negative canonical bundle. J. Algebraic Geom. 1 (1992), no. 2, 279-291.

[244] M. Reid, The moduli space of 3-folds with $K=O$ may nevertheless be irreducible. Math. Ann. 278 (1987), no. 1-4, 329-334.

[245] S.-S. Roan and S.-T. Yau, On Ricci flat 3-fold. Acta Math. Sinica (N.S.) 3 (1987), no. 3, 256-288.

[246] W.-D. Ruan, Canonical coordinates and Bergmann metrics. Comm. Anal. Geom. 6 (1998), no. 3, 589-631.

[247] W.-D. Ruan, Lagrangian torus fibration of quintic hypersurfaces. I. Fermat quintic case. In: Winter School on Mirror Symmetry, Vector Bundles and Lagrangian Submanifolds (Cambridge, MA, 1999), AMS/IP Stud. Adv. Math., 23, Amer. Math. Soc., Providence, RI, 2001, 297-332.

[248] W.-D. Ruan, Lagrangian torus fibration of quintic Calabi-Yau hypersurfaces. II. Technical results on gradient flow construction. J. Symplectic Geom. 1 (2002), no. 3, 435-521.

[249] Y.-B. Ruan, Gromov-Witten invariants and quantum cohomology. In: Geometric Analysis and Applications to Quantum Field Theory (Adelaide, 1998/1999), Progr. Math., 205, Birkhuser Boston, Boston, MA, 2002, 137-156.

[250] Y.-B. Ruan and G. Tian, A mathematical theory of quantum cohomology. J. Differential Geom. 42 (1995), no. 2, 259-367.

[251] J. Sacks and K. Uhlenbeck, The existence of minimal immersions of 2-spheres. Annals Math. 113 (1981), 1-24.

[252] S. Sasaki, On differentiable manifolds with certain structures which are closely related to almost contact structure. Tôhoku Math. J. 2 (1960), 459-476.

[253] R. Schoen and J. Wolfson, Minimizing volume among Lagrangian submanifolds. In: Differential Equations: La Pietra 1996, Proc. of Symp. in Pure Math., vol. 65, 1999, 181-199.

[254] P. Seidel, Homological mirror symmetry for the quartic surface. arXiv:math.SG/ 0310414.

[255] B. Siebert, Symplectic Gromov-Witten invariants. In: New Trends in Algebraic Geometry (Warwick, 1996, London Math. Soc. Lecture Note Ser., 264, Cambridge Univ. Press, Cambridge, 1999), 375-424.

[256] B. Siebert, Algebraic and symplectic Gromov-Witten invariants coincide, Ann. Inst. Fourier (Grenoble) 49 (1999), no. 6, 1743-1795.

[257] Y.-T. Siu, A simple proof of the surjectivity of the period map of K3 surfaces. Manuscripta Math. 35 (1981), 311-321.

[258] Y.-T. Siu, Every K3 Surface is Kähler. Invent. Math. 73 (1983), 139-150.

[259] J. Solomon, Intersection theory on the moduli space of holomorphic curves with Lagrangian boundary conditions. arXiv:math/0606429.

[260] Y.-T. Siu and S.-T. Yau, Compact Kähler manifolds of positive bisectional curvature. Invent. Math. 59 (1980), 189-204.

[261] K. Smoczyk and M.-T. Wang, Mean curvature flows of Lagrangians submanifolds with convex potentials. J. Differential Geom. 62 (2002), no. 2, 243-257.

[262] I. Smith, R. P. Thomas and S.-T. Yau, Symplectic conifold transitions. J. Differential Geom. 62 (2002), 209-242.

[263] M. B. Stenzel, Ricci-ßat metrics on the complexication of a compact rank one symmetric space. Manuscripta Math. 80 (1993), 151-163. 
[264] A. Strominger, Superstrings with torsion. Nucl. Phys. B 274 (1986), 253-284.

[265] A. Strominger, S.-T. Yau and E. Zaslow, Mirror symmetry is T-duality. Nucl. Phys. B 479 (1996), no. 1-2, 243-259.

[266] R. Taylor and A. Wiles, Ring-theoretic properties of certain Hecke algebras. Ann. of Math. (2) 141 (1995), no. 3, 553-572.

[267] R. Thomas, A holomorphic Casson invariant for Calabi-Yau 3-folds, and bundles on K3 fibrations. J. Differential Geom., 54 (2000), 367-438.

[268] R. Thomas and S.-T.Yau, Special Lagrangians, stable bundles and mean curvature flow. Comm. Anal. Geom. 10 (2002), 1075-1113.

[269] G. Tian, Smoothness of the universal deformation space of compact Calabi-Yau manifolds and its Petersson-Weil metric. In: Mathematical Aspects of String Theory (San Diego, Calif., 1986), Adv. Ser. Math. Phys., 1, World Sci. Publishing, Singapore, 1987, 629-646.

[270] G. Tian, On a set of polarized Kähler metrics on algebraic manifolds. J. Differential Geom. 32 (1990), no. 1, 99-130.

[271] G. Tian and S.-T. Yau, Complete Kähler manifolds with zero Ricci curvature. I. J. Amer. Math. Soc. 3 (1990), no. 3, 579-609.

[272] G. Tian and S.-T. Yau, Complete Khler manifolds with zero Ricci curvature. II. Invent. Math. 106 (1991), no. 1, 27-60.

[273] A. Todorov, Applications of the Kähler-Einstein-Calabi-Yau metric to moduli of K3 surfaces. Invent. Math. 61 (1980), no. 3, 251-265.

[274] A. Todorov, The Weil-Petersson geometry of the moduli space of $S U(n)($ CalabiYau) manifolds. I. Comm. Math. Phys. 126 (1989), no. 2, 325-346.

[275] A. Todorov, Weil-Petersson volumes of the moduli spaces of CY manifolds. Comm. Anal. Geom. 15 (2007), no. 2, 407-434.

[276] V. Tosatti, B. Weinkove and S-T. Yau, Taming symplectic forms and the Calabi-Yau equation. Proc. London Math. Soc. 97 (2008), no. 2, 401-424.

[277] K. Uhlenbeck and S.-T. Yau, On the existence of Hermitian-Yang-Mills connections in stable vector bundles. Comm. Pure Appl. Math. 39 (1986), no. S, suppl., S257-S293.

[278] C. Vafa, Extending mirror conjecture to Calabi-Yau with bundles. arXiv:hep-th/ 9804131.

[279] E. Viehweg, Quasi-projective moduli for polarized manifolds. In: Ergebnisse der Mathematik und ihrer Grenzgebiete (3) [Results in Mathematics and Related Areas (3)], 30. Springer-Verlag, Berlin, 1995.

[280] J. Walcher, Opening mirror symmetry on the quintic. Comm. Math. Phys. 276 (2007), no. 3, 671-689.

[281] C.T.C. Wall, Classification Problems in Differential Topology, V: On certain 6-Manifolds, Invent. Math. 1 (1966), 355.

[282] D. Wan, Mirror symmetry for zeta functions. AMS/IP Stud. Adv. Math., 38, Mirror Symmetry. V, Amer. Math. Soc., Providence, RI, 2006, 159-184.

[283] X.-J. Wang and X. Zhu, Kähler-Ricci solitons on toric manifolds with positive first Chern class. Adv. Math. 188 (2004), no. 1, 87-103.

[284] A. Wiles, Modular elliptic curves and Fermat's last theorem. Ann. of Math. (2) 141 (1995), no. 3, 443-551.

[285] P.M.H. Wilson, Calabi-Yau manifolds with large Picard number. Invent. Math. 98 (1989), no. 1, 139-155.

[286] E. Witten, On the structure of the topological phase of two-dimensional gravity. Nucl. Phys. B 340 (1990), no. 2-3, 281-332.

[287] E. Witten, Topological sigma models. Commun. Math. Phys. 118, (1988), 411-449. 
[288] E. Witten, Two-dimensional gravity and intersection theory on moduli space. In: Surveys in Differential Geometry (Cambridge, MA, 1990), Lehigh Univ., Bethlehem, PA, 1991, 243-310.

[289] E. Witten, Phases of $N=2$ theories in two dimension. Nucl. Phys. B403 (1993), 159-222.

[290] B. Weinkove, The Calabi-Yau equation on almost-Kähler four-manifolds, J. Differential Geom. 76 (2007), no. 2, 317-349.

[291] B. Wu, The number of rational curves on K3 surfaces. Asian J. Math. 11 (2007), no. 4, 635-650.

[292] S. Yamaguchi and S-T. Yau, Topological string partition functions as polynomials. J. High Energy Phys. 0407 (2004), 047.

[293] S.-T. Yau, Calabi's conjecture and some new results in algebraic geometry. Proc. Natl. Acad. Sci. USA, 74 (1977), no. 5, 1798-1799.

[294] S.-T. Yau, A general Schwarz lemma for Kähler manifolds. Amer. J. Math. 100 (1978), no. 1, 197-203.

[295] S.-T. Yau, On Ricci curvature of a compact Kähler manifold and complex Monge-Ampère equation I. Comm. Pure and App. Math. 31 (1979), 339-411.

[296] S.-T. Yau, Compact three dimensional Kähler manifolds with zero Ricci curvature. In: Symposium on Anomalies, Geometry, and Topology, (Chicago, IL., 1985), World Sci. Publishing, Singapore, 1985, 395-406.

[297] S.-T. Yau, Nonlinear analysis in geometry. Enseignement Math. 33 (1986) 109-158.

[298] S.-T. Yau and E. Zaslow, BPS states, string duality, and nodal curves on K3. Nucl. Phys. B 471 (1996), no. 3, 503-512.

[299] R. Ye, Gromov's compactness theorem for pseudo holomorphic curves. Trans. Amer. Math. Soc. 342 (1994), no. 2, 671-694.

[300] S. Zelditch, Szegö kernels and a theorem of Tian. Int. Math. Res. Not. 6 (1998), $317-331$.

[301] S. Zhang, Heights and reductions of semi-stable varieties. Compositio Math. 104 (1996), no. 1, 77-105.

[302] I. Zharkov, Torus fibrations of Calabi-Yau hypersurfaces in toric varieties. Duke Math. J. 101 (2000), no. 2, 237-257.

[303] A. Zinger, Enumerative vs. symplectic invariants and obstruction bundles. J. Symplectic Geom. 2 (2004), no. 4, 445-543.

[304] A. Zinger, The reduced genus-one Gromov-Witten invariants of Calabi-Yau hypersurfaces, arXiv:math/0705.2397. 